Journal for ImmunoTherapy of Cancer

\section{Paralysis of the cytotoxic granule machinery is a new cancer immune evasion mechanism mediated by chitinase 3-like-1}

To cite: Darwich A, Silvestri A, Benmebarek M-R, et al. Paralysis of the cytotoxic granule machinery is a new cancer immune evasion mechanism mediated by chitinase 3-like-1. Journal for ImmunoTherapy of Cancer 2021;9:e003224. doi:10.1136/jitc-2021-003224

- Additional supplemental material is published online only. To view, please visit the journal online (http://dx.doi.org/10. 1136/jitc-2021-003224).

Accepted 02 November 2021

Check for updates

(c) Author(s) (or their employer(s)) 2021. Re-use permitted under CC BY-NC. No commercial re-use. See rights and permissions. Published by BMJ.

For numbered affiliations see end of article.

Correspondence to Dr Maria Rescigno; maria.rescigno@hunimed.eu

\section{ABSTRACT}

Background Natural killer (NK) cells require a functional Iytic granule machinery to mediate effective antitumor responses. Evading the lytic cargo deployed at the immune synapse (IS) could be a critical step for cancer progression through yet unidentified mechanisms.

Methods NK cell antibody-dependent cellular cytotoxicity (ADCC) is a major determinant of the clinical efficacy of some therapeutic antibodies including the anti-HER2 Trastuzumab. Thus, we screened sera of Trastuzumabresistant HER2 + patients with breast cancer for molecules that could inhibit NK cell ADCC. We validated our findings in vitro using cytotoxicity assays and confocal imaging of the lytic granule machinery and in vivo using syngeneic and xenograft murine models.

Results We found that sera from Trastuzumab-refractory patients could inhibit healthy NK cell ADCC in vitro. These sera contained high levels of the inflammatory protein chitinase 3-like 1 (CHI3L1) compared with sera from responders and healthy controls. We demonstrate that recombinant CHI3L1 inhibits both $\mathrm{ADCC}$ and innate NK cell cytotoxicity. Mechanistically, CHI3L1 prevents the correct polarization of the microtubule-organizing center along with the lytic granules to the IS by hindering the receptor of advanced glycation end-products and its downstream JNK signaling. In vivo, CHI3L1 administration drastically impairs the control of NK cell-sensitive tumors, while CHI3L1 blockade synergizes with ADCC to cure mice with HER2 +xenografts.

Conclusion Our work highlights a new paradigm of tumor immune escape mediated by CHI3L1 which acts on the cytotoxic machinery and prevents granule polarization. Targeting CHI3L1 could mitigate immune escape and potentiate antibody and cell-based immunotherapies.

\section{INTRODUCTION}

The immune-mediated elimination of cancer cells is dependent on the lytic granule machinery of cytotoxic lymphocytes including $\mathrm{CD}^{+}$cytotoxic $\mathrm{T}$ lymphocytes (CTLs) and Natural killer (NK) cells. While
CTLs require antigen presentation, NK cells are predisposed to respond swiftly, kill transformed cells and regulate the immune response. ${ }^{1} \mathrm{NK}$ cells largely exert their cytotoxic function through the polarized release of cytotoxic mediators stored in lytic granules that is, perforin and granzymes. Granules are released at the NK cell-target cell interface, termed the immune synapse (IS). The lytic IS occurs in a complex and stepwise manner that ensures the efficient elimination of target cells while minimizing bystander killing of healthy cells. ${ }^{2}$ First, on recognition of a potential target, engagement of activation receptors including natural cytotoxicity receptors, Fc receptors (FcR, CD16), and adhesion molecules (ie, LFA-1, Integrins) and/or inhibitory receptors (ie, killer-cell immunoglobulin-like receptors (KIRs)) to their respective ligands will dictate the nature of the IS (inhibitory synapse vs lytic synapse). ${ }^{2}$ NK cells are licensed for cytotoxicity if the activation signals overcome the inhibitory ones. Second, during the effector stage, several key steps take place including actin reorganization and accumulation at the IS, receptor clustering, polarization of the microtubule-organizing center (MTOC) along with the lytic granules to the IS, and lytic-granule fusion with the plasma membrane. Finally, the IS termination stage includes a period of inactivity followed by detachment and potential recycling of cytotoxicity (for serial killing of multiple targets). ${ }^{3}$

Despite the recent advances in our understanding of the biology of the lytic IS and its crucial role in innate and adaptive antitumor immunity, little is known concerning the 
evasion mechanisms that tumor cells can mount at this particular step to ensure cancer progression.

NK cell antibody-dependent cellular cytotoxicity (ADCC) is an important mechanism of action of antitumor therapeutic antibodies. ${ }^{4-8}$ Based on this, we started our project by screening for molecules that were elevated in the sera of trastuzumab-resistant HER2 ${ }^{+}$metastatic patients with breast cancer and that could negatively impact NK cell ADCC. We identified chitinase 3-like 1 (CHI3L1) protein (also known as YKL-40 in humans), as a protein enriched in refractory patients and capable of inhibiting MTOC and granule polarization in NK cells and consequently their innate and ADCC antitumor activity.

CHI3L1 is a mammalian member of the evolutionarily conserved chitinase protein family that still contains a highly conserved chitin-binding domain but lacks chitinase activity due to a mutation in the catalytic site. ${ }^{9}$ Intriguingly, the exact physiological function of YKL-40 is not yet fully characterized but is strongly associated with tissue remodeling and repair according to its expression pattern. ${ }^{10}$ Conversely, there is a consensus on its role as an inflammation-related protein, where its aberrant expression is associated with the pathogenesis of an array of human diseases including asthma, metabolic diseases, and cancer. ${ }^{11}$ In cancer, higher CHI3L1 levels produced by different cell types including macrophages, neutrophils, and tumor cells of different origins have been shown to correlate with a worse prognosis and shorter patient survival in many cancer types. ${ }^{12-17}$ Importantly, CHI3L1 has been described to play a key role in tumor growth and metastasis by inducing several oncogenic properties including angiogenesis, resistance to apoptosis, and tumor invasion. ${ }^{11}$ Despite recent studies correlating CHI3L1 with an immunosuppressive tumor microenvironment (TME), the exact mechanism through which it inhibits the anti-tumor immune response is not known. ${ }^{18}{ }^{19}$ It is also unclear whether high levels of CHI3L1 can inhibit NK cells, hindering their role as sentinels and enabling the development of CHI3L1-mediated metastatic disease. ${ }^{20-25}$ As opposed to their prime role in controlling metastasis formation, NK cells have been generally considered as minor players in the control of solid tumors. ${ }^{26}$ However, this concept has been challenged by the use of ADCC-inducing monoclonal antibodies (eg, Trastuzumab, Cetuximab), immune-checkpoint blockers, NK cell engagers, and chimeric receptors that enhance NK cell cytotoxicity and redirect them to treat solid malignancies. ${ }^{27} 28$

Considering the essential role of the NK cytotoxic granule machinery in translating these immunotherapeutic modalities into tumor cell killing, it would be critical to identify factors that inhibit this process in the TME.

Here, we show that CHI3L1 impairs NK cell cytotoxicity by limiting the polarization of lytic granules and inducing a dysfunctional IS. Our data also support the therapeutic potential of blocking CHI3L1 as a treatment for cancer by enhancing antitumor immunity.
RESULTS

\section{Sera of breast cancer patients with elevated CHI3L1 levels inhibit healthy NK cell activity}

NK cell ADCC is pivotal for the clinical activity of Trastuzumab. ${ }^{5629}$ We postulated that soluble inhibitors of NK cell cytotoxicity could be identified in trastuzumab-treated breast cancer patients by comparing sera from responders to sera from non-responder patients. To this aim, we tested sera from patients with inoperable locally advanced or metastatic HER2 ${ }^{+}$breast cancer that received Trastuzumab plus chemotherapy. We assessed whether these sera could modulate the ADCC activity of healthy NK cells (isolated from PBMCs of healthy donors), against trastuzumab-coated SKBR3 cells. We found that sera from non-responders (ie, that underwent disease progression, $\mathrm{PD}$ ) reduced NK cell ADCC activity while those from responders (either with stable disease (SD) or complete response (CR)) did not affect NK cell ADCC activity (figure 1A). To identify the molecules responsible for this inhibition, we first performed comparative proteomic analysis using the Proteome Profiler Array (R\&D systems) to measure the levels of 102 human soluble proteins (cytokines, chemokines, and growth factors) (not shown). We selected the most differentially expressed hits to be included in a multiplex Luminex immunoassay (R\&D systems) panel of 12 proteins: GDF15, VitD-BP, RBP4, BDNF, CHI3L1, ICAM-1, IGFBP-3, Angiogenin, sCD14, CRP, Cystatin, and DPPIV. We confirmed that some of these candidates were differentially expressed between healthy donors and patients (whether responders or not) (online supplemental figure S1). These included the previously identified breast cancerassociated molecules with prognostic value such as sCD14 and GDF15. ${ }^{30}$ Cystatin $\mathrm{C}$ levels were also increased which might indicate a worsening of renal function in these heavily treated patients. ${ }^{32}$ However, among these, only CHI3L1 levels could distinguish between sera from non-responders (PD) and responders $(\mathrm{CR}+\mathrm{SD})$ (online supplemental figure $\mathrm{S} 1)$.

Interestingly, changes in CHI3L1 levels during Trastuzumab treatment correlated with the inhibitory effects of the sera. In responders, serum CHI3L1 levels were unchanged and comparable to levels observed in healthy controls. Conversely, basal CHI3L1 levels were higher in the sera of some PD patients and increased even further at the time of PD (figure 1B). To validate our findings from this discovery cohort recruited before the introduction of another anti-HER2 antibody (pertuzumab), we recruited a second cohort of patients receiving the current standard therapy for HER2 ${ }^{+}$inoperable locally advanced or metastatic breast cancer (trastuzumab+pertuzumab+chemotherapy). In this cohort, 'basal' blood samples were taken during therapy when patients were in SD, and an 'endpoint' sample following a clinical outcome (PD, CR, or remained in SD after 18 months). In accordance with the previous cohort, PD patient sera had an inhibitory effect on healthy NK cell ADCC (figure 1C) and contained higher levels of CHI3L1 compared with sera of CR patients and healthy subjects (figure 1D,E). Importantly, a neutralizing antibody directed to human CHI3L1 significantly reduced the inhibitory effect of PD patients' sera on healthy NK cell ADCC (figure 1F). Hence, CH3L1 is increased in sera of 
- Responders (CR+SD) ○ Non responders (PD)
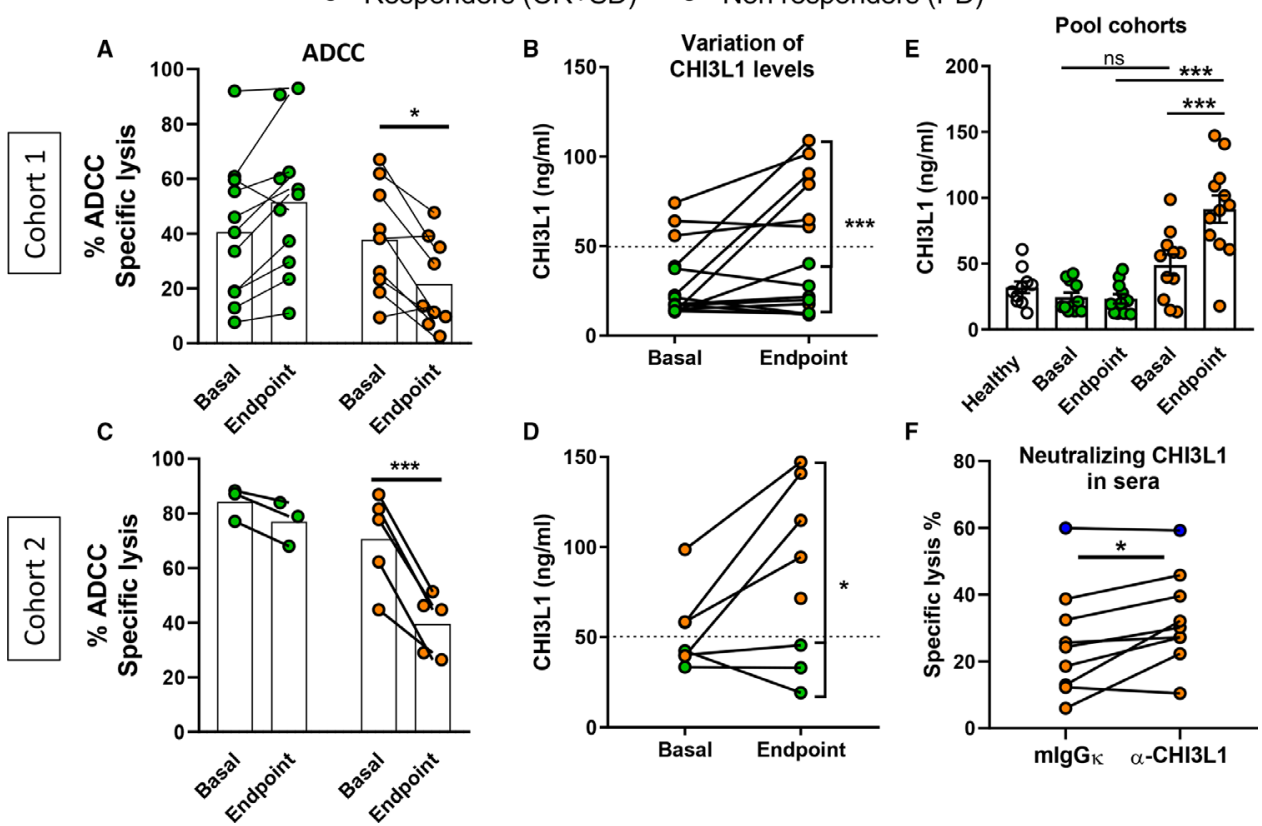

Figure 1 Sera of trastuzumab-resistant patients negatively modulate NK cell activity and contain high levels of CHI3L1 protein. Blood samples were collected at base (before trastuzumab therapy in cohort 1 and during response to therapy in cohort 2) and when patients reached progressive disease (PD), complete response (CR), or stable disease (SD) within 18 months' follow-up. (A, C) ADCC-specific lysis of SKBR3 tumor cells by preactivated (200 UI/mL IL-2 O.N) healthy NK cells treated with sera of responders $(N=14)$ or non-responders $(N=14)(B, D)$ variation of serum $C H I 3 L 1$ levels from base to clinical outcome in cohort 1 and 2, respectively. (E) CHI3L1 levels at base and at clinical endpoint in patients from the two cohorts ( $N=10$ healthy, $\mathrm{N}=14$ responders and N=14PD). (F) ADCC-specific lysis of SKBR3 targets in the presence of Isotype control (IgGk) or anti-CHI3L1 neutralizing antibody to inhibit the effects of CHI3L1 from PD patients' sera ( $\mathrm{N}=8$ in orange with statistics, and autologous healthy serum in blue). Values represent the means \pm SEM $\left(95 \% \mathrm{Cl}{ }^{\star} \mathrm{p}<0.05,{ }^{* \star} \mathrm{p}<0.01,{ }^{\star \star *} \mathrm{p}<0.001\right.$, ns, not significant). ADCC, antibody-dependent cellular cytotoxicity; CHI3L1, chitinase 3-like 1; NK, natural killer.

Trastuzumab-treated PD patients and is responsible for inhibiting NK cell activity. To extend the relevance of our results to larger patient cohorts, we analyzed publicly available gene expression datasets. We found that a higher gene expression of CHI3L1 in primary tumors correlated with worse overall survival and distant metastasis-free survival in $\mathrm{HER}^{+}$breast cancer (online supplemental figure S2A,B). Similarly, higher expression levels also correlated with worse progression-free survival in HER2 ${ }^{+}$gastric cancer, where trastuzumab is also a therapeutic option (online supplemental figure S2C). Interestingly, CHI3L1 was also overexpressed in primary tumors of cetuximab-resistant metastatic colorectal cancer patients (monotherapy that is also dependent on NK cell activity for efficacy) ${ }^{33} 34$ (online supplemental figure S2D).

\section{CHI3L1 inhibits NK cell cytotoxicity in vitro}

To test if CHI3L1 could directly affect NK cell cytotoxicity, we pretreated healthy NK cells with different concentrations of CHI3L1 before incubating them with target cells. We found that the addition of CHI3L1 impaired both antibody-dependent and innate cytotoxicity of purified human and murine NK cells (figure 2A-D). We confirmed the involvement of CHI3L1 in the observed phenomenon by adding an anti-human CHI3L1 neutralizing antibody to the test, which abrogated the inhibitory effect of rhCHI3L1 in a dose-dependent manner (figure 2E). Intriguingly, CHI3L1 treatment did not affect the amount of NK cells degranulation as measured by CD107a staining (figure 2F) nor did the expression of the cytotoxicity receptors, CD16 and NKG2D, that mediate ADCC and natural cytotoxicity, respectively (figure 2G,H). Similarly, the expression of a panel of other receptors involved in modulating NK cell cytotoxicity, such as DNAM-1, NKG2A, CD11a (LFA-1), CD69, NKp30, NKp46, CD2, KIR2DL1, and KIR2DL2/L3, was unaffected by CHI3L1 treatment (online supplemental figure S3A). Interestingly, we observed a similar CHI3L1dependent inhibition of $\mathrm{CD}^{+} \mathrm{T}$ cell cytotoxicity against MEC-1 targets loaded with Staphylococcus enterotoxin B (SEB) super-antigen without affecting CD107 expression (online supplemental figure S3B,C). Together, these results indicate that CHI3L1 can inhibit the cytotoxic activity of NK cells (both natural and ADCG).

\section{CHI3L1 impairs the polarization of the lytic machinery in NK cells}

The inhibitory effect exerted by CHI3L1 on both, ADCC and natural cytotoxicity of NK cells, was independent of modulating cytotoxicity receptors or the amount of degranulation. Thus, we tested whether CHI3L1 might be acting on the lytic granules' machinery, a common pathway of both killing modalities. In NK cells, the IS is formed in a stepwise and controlled manner: they first form conjugates with target cells, inducing a signal for 
A

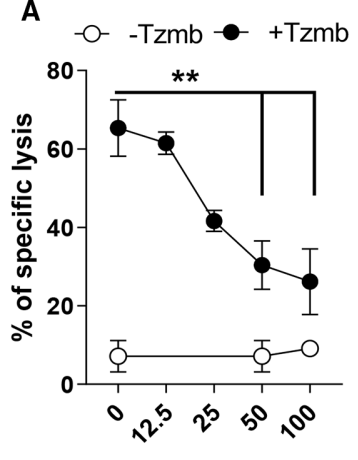

[CHI3L1] ng/ml

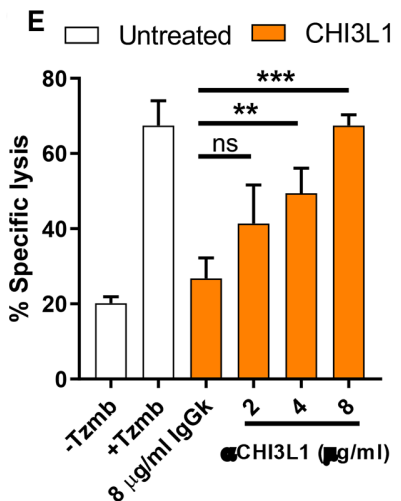

B

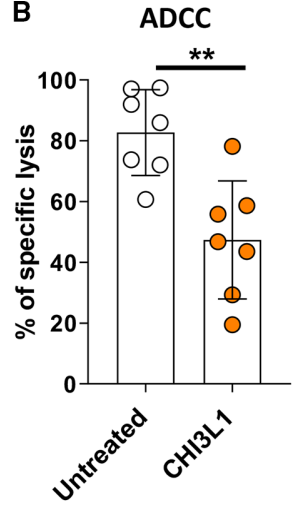

F $\quad$ CD107a

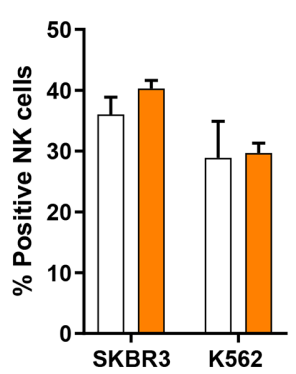

C

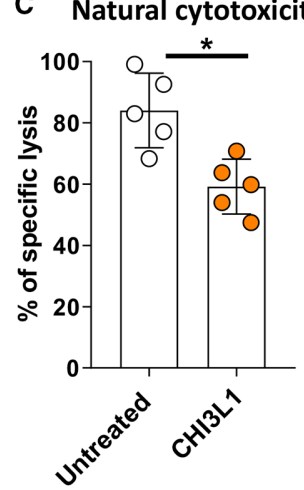

D

Murine NKs ADCC
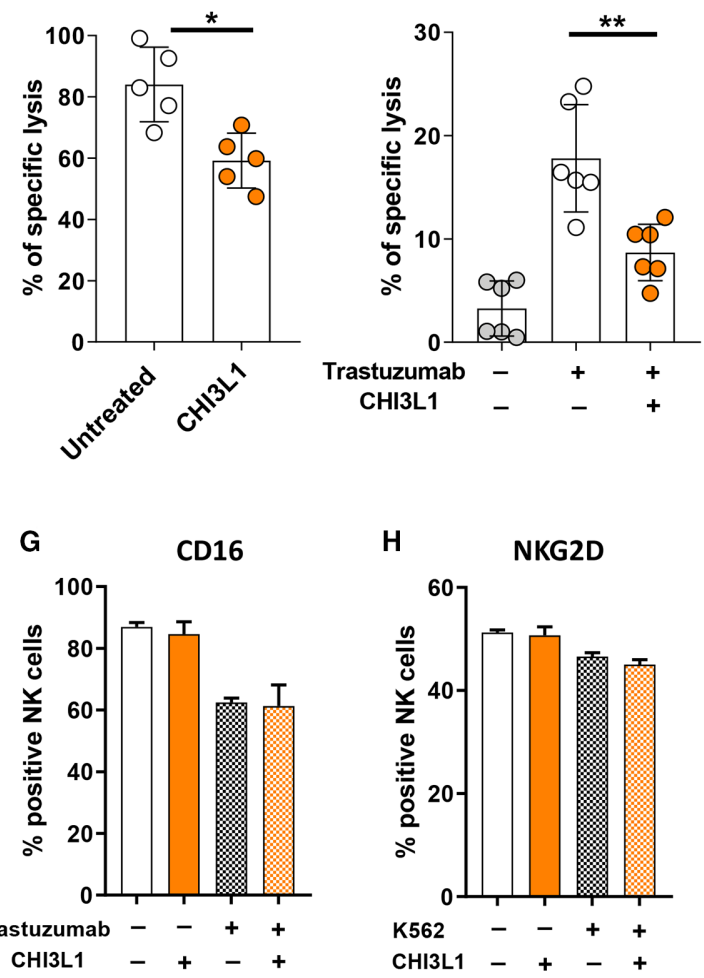

Figure 2 CHI3L1 inhibits ADCC-dependent and natural cytotoxicity of NK cells. (A) ADCC-specific lysis of SKBR3 tumor cells by human NK cells preactivated O.N with IL-2 $(200 \mathrm{UI} / \mathrm{mL})$ and treated with increased dose of rhCHI3L1 (B) Pooled data (NK cells isolated from $\mathrm{N}=7$ healthy donors) treated with rhCHI3L1 $(100 \mathrm{ng} / \mathrm{mL})$ for 1 hour before ADCC-specific lysis of SKBR3 tumor cells assay. (C) NK cell natural cytotoxicity against K562 targets after treatment with rhCHI3L1 (100 ng/mL) (NK cells from $\mathrm{N}=5$ healthy donors). (D) ADCC-specific lysis of SKBR3 cells by preactivated splenic murine NK cells treated with recombinant mouse rmCHI3L1 (100 ng $/ \mathrm{mL}$ ) (NK cells from $n=6 B A L B / c$ mice). (E) ADCC-specific lysis of SKBR3 targets in the presence of anti-CHI3L1 neutralizing antibody or Isotype control (lgGk) to inhibit the effects of rhCHI3L1. (F, G, H) Flow cytometry analysis of the frequencies of CD107a, CD16 and NKG2D-positive NK cells alone or following incubation with target cells: SKBR3 (ADCC) or K562 (natural cytotoxicity), with or without rhCHI3L1 $(100 \mathrm{ng} / \mathrm{mL})$. All plots are representative of three independent evaluations. Values in all graphs represent the means $\pm \mathrm{SD}\left(95 \% \mathrm{Cl},{ }^{*} \mathrm{p}<0.05,{ }^{* \star} \mathrm{p}<0.01,{ }^{* \star *} \mathrm{p}<0.001\right.$, ns, not significant). ADCC, antibody-dependent cellular cytotoxicity; CHI3L1, chitinase 3-like 1; NK, natural killer.

cytoskeletal rearrangement that leads to the formation of a dense F-actin mesh at the IS. The granules then accumulate at the MTOC before jointly polarizing towards the target cell surface. ${ }^{2}$ We used confocal microscopy to study these major steps and found that CHI3L1 treatment impaired perforin polarization to the IS despite the correct accumulation of F-actin and LFA-1, two early requirements of correctly formed lytic conjugates (figure 3A,D). We next analyzed MTOC polarization and found that despite an observed perforin accumulation at the MTOC, NK cells failed to polarize their MTOC and perforin to the IS (figure 3A,D). These findings were confirmed by live-cell imaging where we observed efficient target cell engagement by NK cells followed by the accumulation of lytic granules at the MTOC, before an eventual detachment without a productive release of lytic granules towards the target cells (figure 3C, online supplemental movies 1 and 2). To further elucidate the effect of CHI3L1 on the lytic machinery we treated healthy NK cells and evaluated their cytotoxic granule content. We found that CHI3L1 treatment decreased the levels of perforin, granzyme A, and granzyme B in NK cells in a time and dose-dependent manner, even in the absence of a target cell (figure 3E and online supplemental figure S4). This activity was reversed by adding an anti-CHI3L1 antibody (online supplemental figure S4). These results indicate that CHI3L1 does not affect the formation of the IS but inhibits the correct polarization of the MTOC and consequently of the lytic granules. Further, CHI3L1 favors the loss of granules even in the absence of an activation signal.

\section{CHI3L1 hinders receptor of advanced glycation end-products signaling in NK cells}

To elucidate the molecular mechanism of action, we first analyzed the expression of known CHI3L1 receptors on NK cells, including interleukin 13 receptor subunit alpha 2 (IL13R $\alpha 2)$, the receptor of advanced glycation end products (RAGE), and Prostaglandin DP2 receptor (CRTH2). ${ }^{35-37}$ CRTH2 is expressed on a small subset of NK cells, ${ }^{38}$ while IL13R $\alpha 2$ is not expressed on the NK cell surface as shown by flow cytometry and confocal 
A
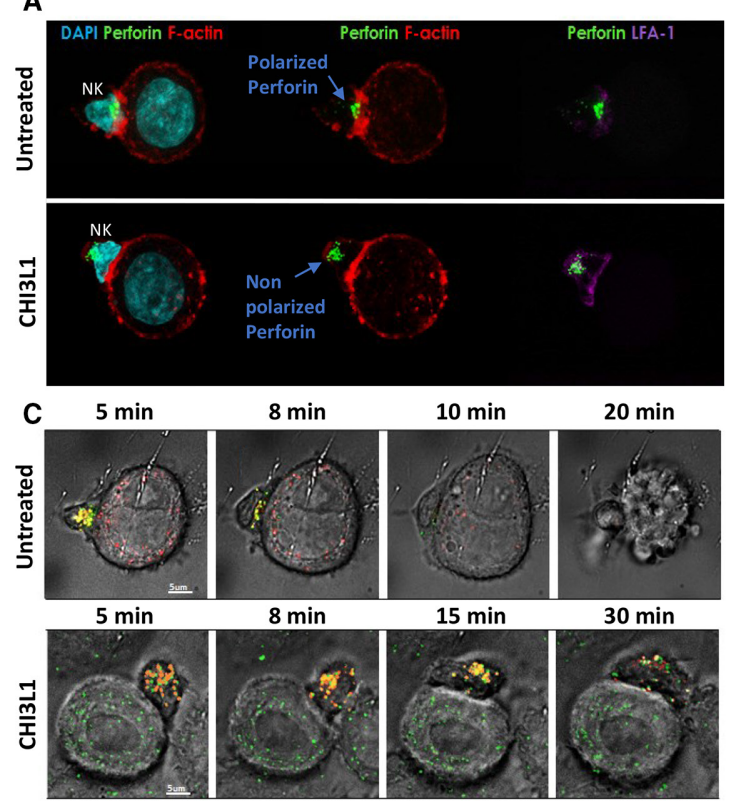

D

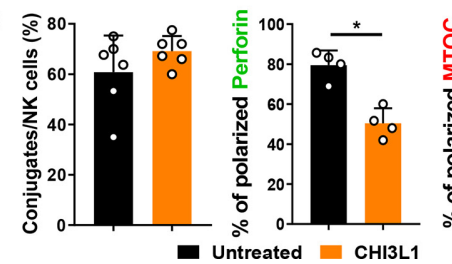

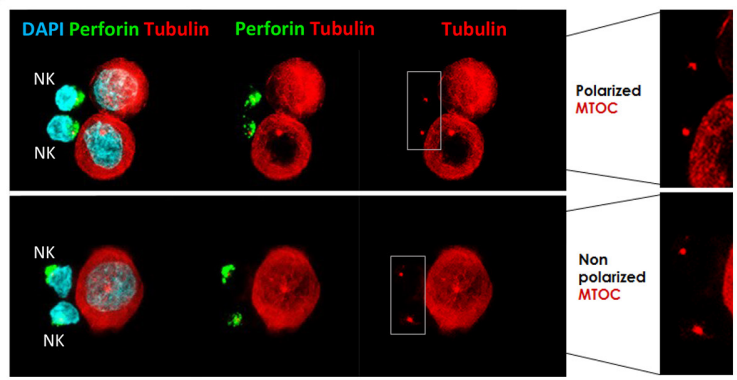
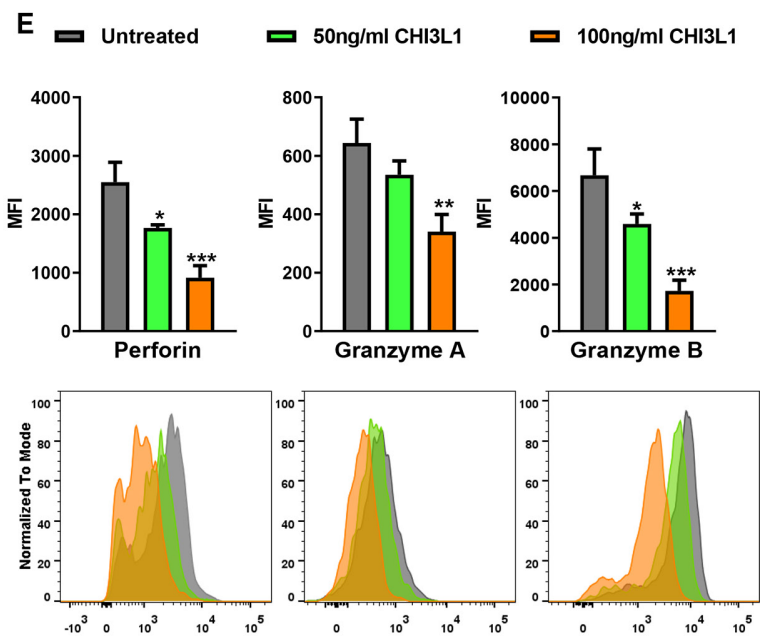

Figure 3 The decreased cytotoxicity of NK cells results from defective MTOC and granule polarization after CHI3L1 treatment. Confocal microscopy images of the immune synapse between IL-2 stimulated (200 UI/mL O.N) human NK cells treated or not with rhCHI3L1 $(100 \mathrm{ng} / \mathrm{mL})$ and SKBR3 cells treated with Trastuzumab. (A) Staining of perforin and early polarization markers (F-actin and LFA-1). (B) Staining for perforin and the MTOC. (C) Live cell imaging of NK cells in ADCC with SKBR3 targets at 2:1 Effector:Target ratio and followed for 2 hours (Red: lysotracker for granules, Green: Sir-tubulin for MTOC) (D) Frequency of NK:Target conjugates (left) frequency of polarized synapses among conjugates (middle and right) (at least 25 conjugates/ treatment/donor were counted from several fields of view within the slide, each dot represents a donor) (E) Flow cytometry analysis of lytic granules perforin, granzyme A and granzyme B in NK cells alone or treated with CHI3L1 for 2 hours. Values represent the means $\pm \mathrm{SD}\left(95 \% \mathrm{Cl},{ }^{\star} \mathrm{p}<0.05,{ }^{* \star} \mathrm{p}<0.01,{ }^{* \star} \mathrm{p}<0.001\right)$. ADCC, antibody-dependent cellular cytotoxicity; CHI3L1, Chitinase 3-like 1; IL-2, interleukin 2; MTOC, microtubule-organizing center; NK, natural killer

imaging (online supplemental figure S5A,B). By contrast, consistent with a previously described activating function of RAGE in NK cells, ${ }^{39} 40$ we found this receptor to be expressed at higher levels on the CD56 ${ }^{\mathrm{dim}} \mathrm{NK}$ cell population (online supplemental figure S5C). Further, the expression of RAGE was downmodulated after incubation with CHI3L1, suggesting that it might be internalized on CHI3L1 binding (figure 4A). We found that CHI3L1 bound to RAGE with a good affinity $\mathrm{Kd} \sim 16 \mathrm{nM}$ (not shown). Importantly, we observed that RAGE accumulated at the IS during ADCC, highlighting a functional involvement of this receptor in regulating the biology of the IS (online supplemental figure S5D). A RAGE blocking antibody, akin to CHI3L1 treatment, induced a decrease of lytic granules (figure 4B), inhibited NK cell cytotoxicity (figure 4C) and impaired MTOC polarization to the IS (figure 4D). These data suggested that CHI3L1 mediates its inhibitory effects in NK cells through negative modulation of RAGE signaling. To address this issue, we evaluated the effect of CHI3L1 ligation on signaling pathways downstream of RAGE. Specific signaling molecules such as ERK1/2, p38 MAPK, SAPK/JNK, and NF- $\kappa B$ are triggered by different ligands/RAGE interactions in different cell types, ${ }^{41}$ but their involvement in NK cells is unknown. We focused on pathways that are important for NK cell cytotoxicity, including granule biology and MTOC polarization, namely ERK1/2, AKT, STAT3, and JNK. ${ }^{42} 43$ While CHI3L1 treatment did not impact ERK1/2, AKT, or STAT3 phosphorylation levels (online supplemental figure S5E), basal P-JNK levels were decreased in a dosedependent manner in CHI3L1-treated healthy NK cells (figure $4 \mathrm{E}$ ). Consequently, we decided to evaluate the effect of CHI3L1 on ligand-induced JNK activation. In particular, we used the RAGE agonist S100A8/A9 (Calprotectin), which has been described to activate murine NK cells through RAGE $^{39}$ and compete with CHI3L1 on RAGE binding. ${ }^{36}$ Indeed, S100A8/A9 rapidly induced JNK phosphorylation whereas pre-incubation of healthy NK cells with CHI3L1 before treatment opposed this activation (online supplemental figure S5F and figure $4 \mathrm{~F}$ ). Besides affecting basal JNK levels and ligand-induced 

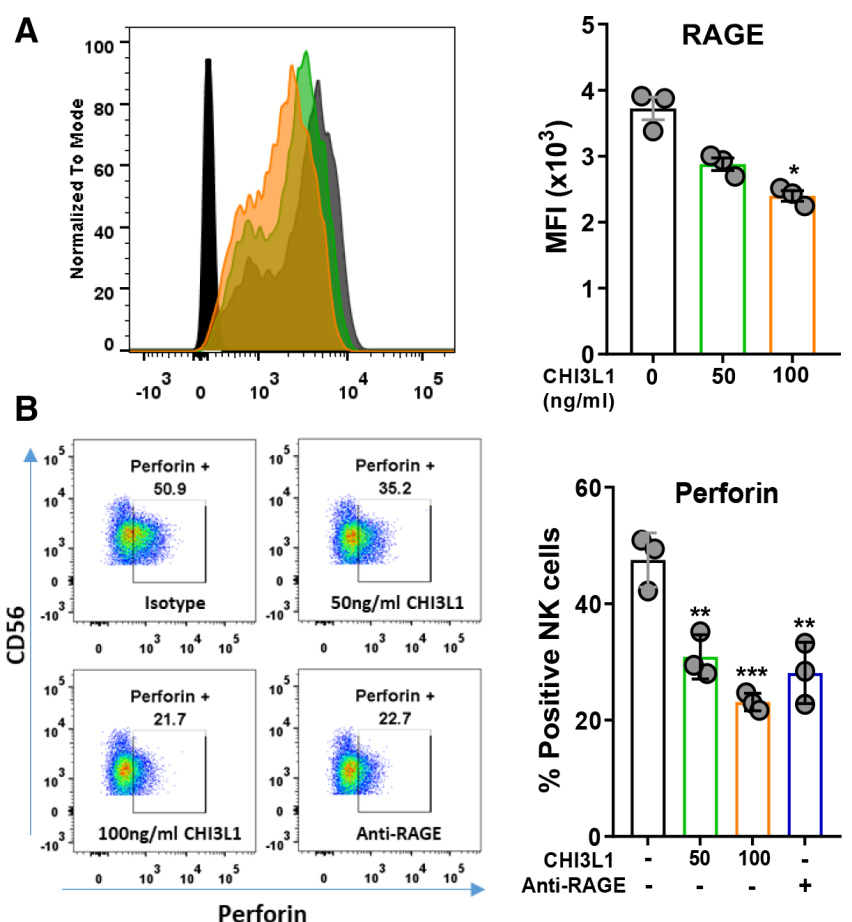

D
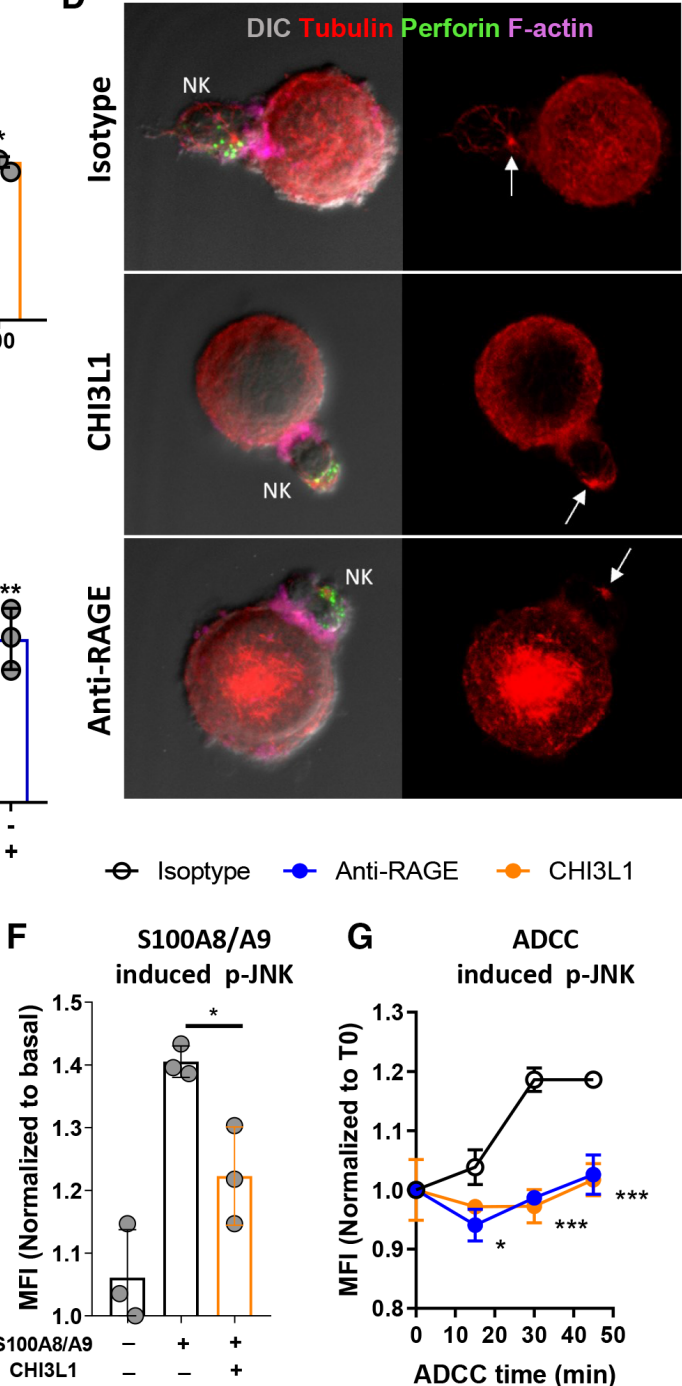

C

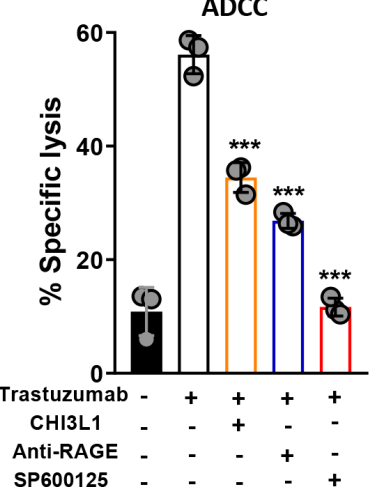

E

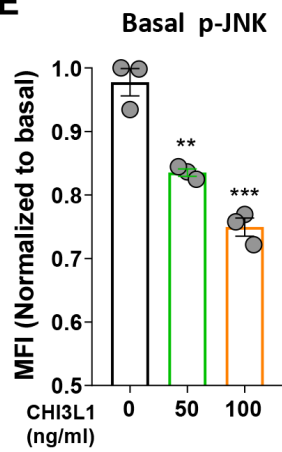

G

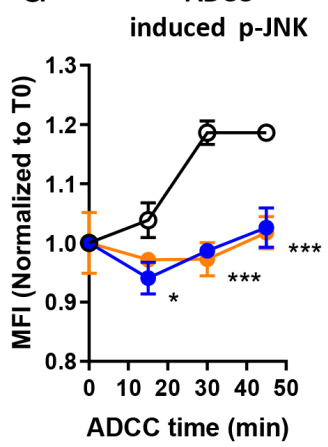

Figure 4 CHI3L1 exerts its effects by inhibiting JNK signaling downstream of RAGE. (A) Flow cytometry staining of RAGE expression on isolated human NK cells pre-activated with IL-2 O.N and treated with increasing doses of rhCHI3L1. (B) Flow cytometry analysis of frequencies of perforin ${ }^{+} \mathrm{NK}$ cells treated with rhCHI3L1 or anti-RAGE blocking antibody $(10 \mu \mathrm{g} / \mathrm{mL})$ for 1 hour. (C) ADCC-specific lysis of SKBR3 tumor cells by NK cells treated with rhCHI3L1, anti-RAGE blocking antibody (10 $\mu$ g/ $\mathrm{mL}$ ) or JNK inhibitor SP600125 (10 uM) for 1 hour before assay. (D) Confocal images of the immune synapse between SKBR3 tumor cells and NK cells treated with rhCHI3L1 or anti-RAGE blocking antibody $(10 \mu \mathrm{g} / \mathrm{mL})$ for 1 hour, stained for perforin, Factin and tubulin. (E) Basal p-JNK levels in IL-2 stimulated NK cells treated with increasing doses of rhCHI3L1. (F) p-JNK levels in NK cells after $10 \mathrm{~min}$ of stimulation by RAGE ligand S100A8/A9 $(10 \mu \mathrm{g} / \mathrm{mL})$ alone or in competition with rhCHI3L1 (100 ng/ $\mathrm{mL})$. (G) p-JNK levels during ADCC of NK cells pretreated with rhCHI3L1 or anti-RAGE for 1 hour and incubated with SKBR3 targets at (5:1) Effector:Target ratio. All plots are representative of at least two independent evaluations. Values represent the means $\pm S D, 95 \% \mathrm{Cl},{ }^{*} \mathrm{p}<0.05,{ }^{* *} \mathrm{p}<0.01,{ }^{* * *} \mathrm{p}<0.001$. ADCC, antibody-dependent cellular cytotoxicity; CHI3L1, Chitinase 3-like 1; IL-2, NK, interleukin 2; natural killer; RAGE, receptor of advanced glycation end-products.

JNK activation, we wanted to assess whether CHI3L1 affected JNK signaling also during NK cell cytotoxicity. As expected, JNK was induced in a time-dependent manner during ADCC (figure 4G). Importantly, pretreatment of healthy NK cells with CHI3L1 or anti-RAGE significantly reduced this induction, suggesting impaired JNK activation during ADCC in CHI3L1 and anti-RAGE treated NK cells (figure 4H). Congruently, treating NK cells with the JNK inhibitor SP600125 inhibited MTOC polarization (online supplemental figure S5G) and strongly impaired their cytotoxicity (figure 4C), consistent with a previous study reporting that JNK is required for MTOC polarization in NK cells. ${ }^{43}$ Together, these results indicate that CHI3L1 binds to RAGE and acts as an inhibitor of its downstream JNK signaling, an essential step for MTOC polarization.

Having observed a contribution of RAGE on granules' polarization in NK cells, we next wanted to investigate its role in vivo, so we used a soluble form of RAGE (sRAGE-Fc) to interfere with membrane-bound RAGE 
and its possible interaction with CHI3L1. We used the 4T1 triple-negative breast cancer model where CHI3L1 levels are known to be elevated and to strongly promote tumorigenesis and metastases. ${ }^{44}$ sRAGE treatment decreased 4T1 primary tumor growth with impressive inhibition of lung metastases formation (online supplemental figure S6A-E). This correlated with a longer survival, decreased CHI3L1 serum levels, and enhanced CD8 + T cell function in sRAGE treated mice (online supplemental figure S6F-H).

Overall, these data suggest that CHI3L1 exerts its in vivo activity at least in part via RAGE, impacting both primary tumor growth and metastasis formation.

\section{In vivo administration of CHI3L1 abrogates NK cell tumor surveillance}

We next investigated the effect of CHI3L1 on tumor control by NK cells in vivo. RMA-S lymphoma cells are TAP2 deficient and thus express low levels of MHC-I molecules on their surface. ${ }^{45}$ This makes them susceptible to NK cell lysis in an NKG2D/perforin-dependent mechanism and an ideal model to study NK cell activity in vivo. ${ }^{46}$ RMA-S cells did not express CHI3L1 (not shown), and their proliferation was not affected by treatment with the murine recombinant protein in vitro (figure $5 \mathrm{~A}$ ). Next, we injected mice with a suboptimal number of RMA-S cells to reveal the effects of CHI3L1 on tumor growth in vivo, followed by treatment with PBS, rmCHI3L1, or anti-NK1.1 antibody (figure 5B). Tumor engraftment and growth were markedly enhanced by rmCHI3L1 injections, to a similar extent as mice treated with an NK cell-depleting antibody (anti-NK1.1), used as a positive control (figure 5C-F). In line with the observed effect on tumor growth, splenocytes from rmCHI3L1-treated or NK cell-depleted mice were less cytotoxic ex vivo against RMA-S cells (figure 5G). Altogether these data indicated that CHI3L1 enhances tumorigenesis of RMA-S cells at least in part by inhibiting NK cell cytotoxicity. To further test this hypothesis, we performed a second experiment in which we treated mice with a combination of NK1.1 antibody and rmCHI3L1 to reveal other NK cell-independent
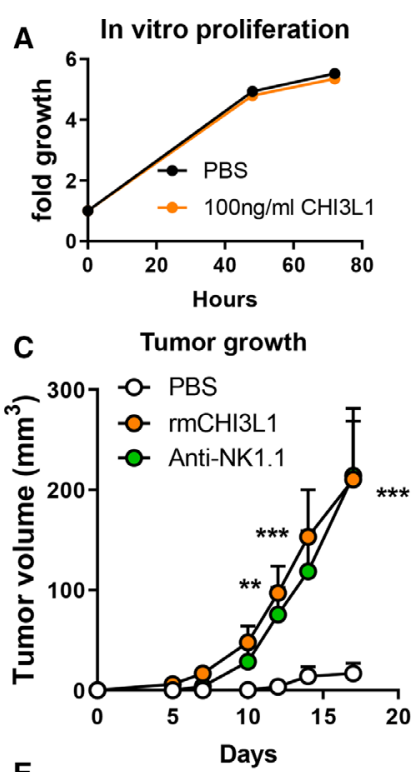

$\mathbf{E}$

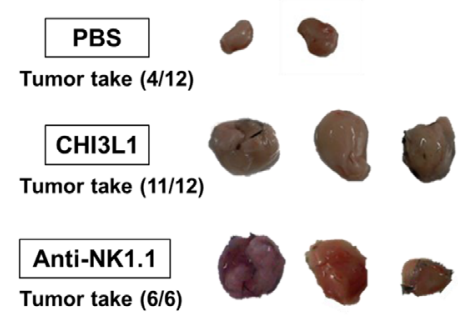

B

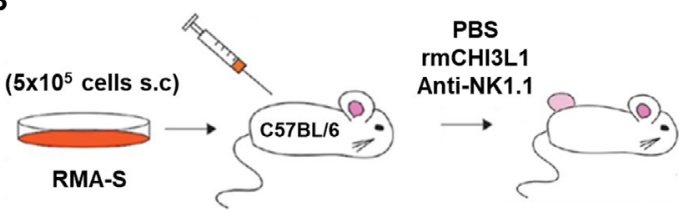

D

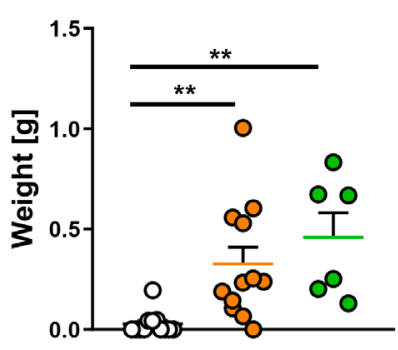

E NK cell Frequency

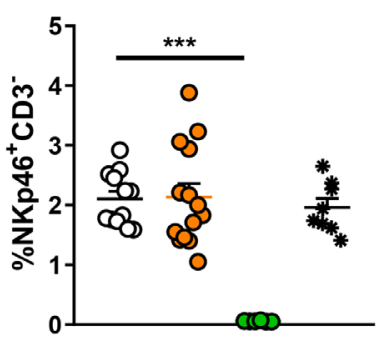

Ex-vivo Cytotoxicity

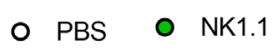

- $\mathrm{CHI} 3 \mathrm{~L} 1 *$ Tumor free
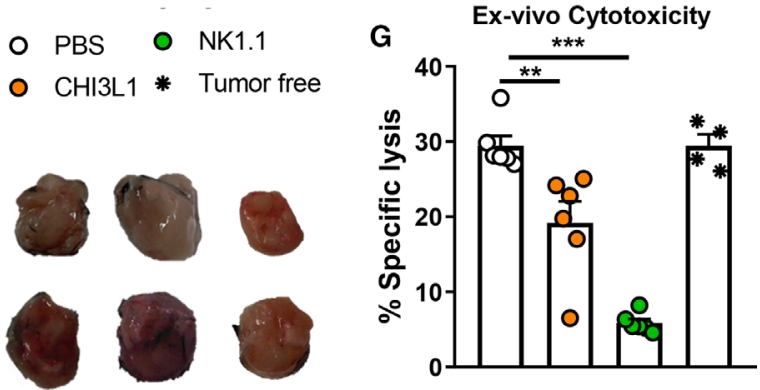

Figure 5 CHI3L1 administration increases tumor engraftment and growth of RMA-S tumors. (A) In vitro CyQUANT cell proliferation assay of RMA-S cells treated or not with rmCHI3L1. (B) RMA-S lymphoma cells were injected s.c in the flank at a limiting number of cells $\left(5 \times 10^{5}\right)$ at day 0 . Mice were randomized in three groups to receive PBS, rmCHI3L1 (0.5 $\mathrm{Mg} / \mathrm{mouse}$ i.p every other day starting from day 0), or anti-NK1.1 (150 $\mu \mathrm{g} /$ mouse on day 0, day 2, and day 4). Tumor growth (C) and weight (D) are represented. (E) Representative images of tumors $\mathrm{N}=2$ experiments. (F) FACS analysis of NK cell frequency in the spleen among total live splenocytes (G) Ex-vivo cytotoxicity assay against RMA-S cells using mouse splenocytes, from describedexperimental groups, cultured O.N with $10 \mathrm{ng} / \mathrm{mL} \mathrm{IL-15}$ and $200 \mathrm{UI} / \mathrm{ml} \mathrm{IL-2} \mathrm{(E:T} \mathrm{40:1).} \mathrm{Data} \mathrm{is} \mathrm{pooled} \mathrm{from} \mathrm{two} \mathrm{independent}$ experiments for PBS and rmCHI3L1 groups ( $\mathrm{N}=12$ mice per group) and one experiment for anti-NK1.1 treated group ( $\mathrm{N}=6$ mice). Values represent the means $\pm \mathrm{SEM}, 95 \% \mathrm{Cl},{ }^{* *} \mathrm{p}<0.01,{ }^{\star \star *} \mathrm{p}<0.001$. CHI3L1, Chitinase 3-like 1; IL-2, interleukin 2; NK, natural killer. 
protumorigenic effects of CHI3L1. We observed that the combination treatment had at least an additive effect on tumor growth (online supplemental figure S7A,B). Interestingly, NK cell depletion was slightly but significantly decreased in the combo group compared with anti-NK1.1 treatment alone (online supplemental figure S7C). This might result from CHI3L1 inhibiting NK cell ADCC which contributes to the effect of depleting antibodies. While NK cell maturation (CD27, CD11b staining) was not affected, expression of cytotoxic granules and T-bet was decreased in NK cells from spleens of CHI3L1-treated mice (online supplemental figure S7C). Similarly, perforin levels were strongly decreased in splenic NKT and CD8 T cells on CHI3L1 treatment (online supplemental figure S7D,E). Analysis of tumor-immune infiltrates revealed a remarkable increase of macrophages and a decrease of $\mathrm{T}$ cells (both CD4 and CD8) on CHI3L1 treatment (online supplemental figure S7F). CD8 T cells were also less active in the tumor as suggested by decreased CD44 expression while CD4 T cells had increased IFN-g and CD25 expression which might indicate a regulatory phenotype. ${ }^{47}$

Overall, data from this model indicate that CHI3L1 suppresses anti-tumor immunity while potentially favoring tumor-promoting immune cells.

\section{CHI3L1 overexpression in tumor cells impairs ADCC efficacy in vivo}

NK cell ADCC is known to be a major determinant of in vivo efficacy of the anti-HER2 antibody trastuzumab. ${ }^{5629}$ To study the effect of CHI3L1 on this important function, we used a HER2 ${ }^{+}$breast cancer xenograft model to allow us to evaluate whether CHI3L1 overexpression could lead to trastuzumab resistance. In particular, we used human JIMT-1 cells which are intrinsically resistant to HER2 blockade by trastuzumab in vitro. However, JIMT-1 tumor growth can still be partially controlled in vivo through NK cell ADCC when trastuzumab treatment is started at a tumor volume of around $50-100 \mathrm{~mm}^{3}{ }^{3.8}$ Thus, a loss of Trastuzumab efficacy in this model directly reflects the ability of NK cells to mediate efficient ADCC, without other confounding factors such as the inhibition of HER2 ${ }^{+}$signaling. To study the effect of CHI3L1 in this system, we transfected JIMT-1 cells, that do not express human CHI3L1 (not shown), to stably overexpress the murine CHI3L1 protein. Overexpression of mCHI3L1 did not affect the proliferation of JIMT-1 cells in vitro (online supplemental figure S8A).

In the first set of experiments, we injected nude mice with JIMT-1-Mock and JIMT-1-mCHI3L1 cells to evaluate the effect of expressing mCHI3L1 on tumor growth in vivo (online supplemental figure $\mathrm{S} 8 \mathrm{~B}$ ). We used inbred $(\mathrm{BALB} / \mathrm{c})$ and outbred $(\mathrm{CD} 1)$ nude mice to evaluate whether CHI3L1 effects are strain dependent. Tumor growth was increased in mice injected with JIMT-1mCHI3L1 cells regardless of the genetic background, although $\mathrm{CD} 1$ nude mice were more resistant than $\mathrm{BALB} / \mathrm{c}$ nude mice to tumor development (only tumor weight was statistically different in CD1 mice) (online supplemental figure $\mathrm{S} 8 \mathrm{C}, \mathrm{D})$. This was paralleled by higher mCHI3L1 levels in the sera of mice with mCHI3L1 overexpressing tumors (online supplemental figure S8E). Interestingly, in $\mathrm{CD} 1$ mice some mock tumors completely regressed $(3 / 5)$ while all mCHI3L1 overexpressing ones continued to grow. When we analyzed NK cells (the main cytotoxic effectors in nude mice) we found that, while their frequency was unaltered in CD1 mice injected with CHI3L1 tumors, their activation status was affected when compared with NK cells from mock tumor-bearing mice, as indicated by reduced NKG2D and CD69 expression (online supplemental figure S8F).

In the second set of experiments, we proceeded with our main goal to assess the effect of CHI3L1 expression on the efficacy of Trastuzumab (ADCC) in controlling tumor growth. We used BALB/c nude mice for these experiments being more permissible for JIMT-1 tumor growth. We first confirmed that HER2 expression levels were comparable between mock and mCHI3L1overexpressing cells (figure 6A). We injected BALB/c nude mice with different doses of JIMT-1 mock and JIMT1-mCHI3L1 cells to synchronize the differential tumor growth kinetics observed in the previous experiment and to reduce the time gap before starting trastuzumab treatment (figure 6B). As reported, ${ }^{48}$ trastuzumab partially inhibited the growth of JIMT-1 tumors (figure 6C). However, this effect was abrogated with mCHI3L1 overexpression whereby tumors were rendered completely insensitive to trastuzumab injections (figure 6C-E). This was paralleled by higher mCHI3L1 sera levels in these mice (figure 6F). In line with the differences in trastuzumab efficacy in vivo, we observed decreased ex vivo ADCC of splenocytes isolated from mice injected with mCHI3L1-overexpressing tumors against JIMT-1 targets compare to the control group (figure 6G). We observed no differences in the expression of the degranulation marker CD107a (figure $6 \mathrm{H}$ ). This is in accordance with our observation on human NK cells, where CHI3L1 affects granule polarization rather than the degranulation itself.

Last, we wanted to characterize immune differences between CHI3L1 overexpressing tumors and Mock tumors. We sacrificed mice during response (Mock tumors) or no response (CHI3L1 tumors) after the second cycle of trastuzumab (online supplemental figure S9A,B). Similarly, to the RMA-S tumor model, we observed higher number of Macrophages in CHI3L1 overexpressing tumors (online supplemental figure S9C). Of note, trastuzumab treatment equally increased CD11b expression on macrophages from Mock and CHI3L1 tumors (online supplemental figure S9C). NK cell number and phenotype were unchanged in the spleens (online supplemental figure S9D). In the tumor, NK cell infiltration was strongly increased in trastuzumab treated mice, indicating engagement of these cells in ADCC (online supplemental figure S9E). Paradoxically, the frequency of CD11b+NK cells (mature NK cells) was decreased by Trastuzumab treatment although to a larger extent in CHI3L1 overexpressing tumors (online supplemental figure S9E). 

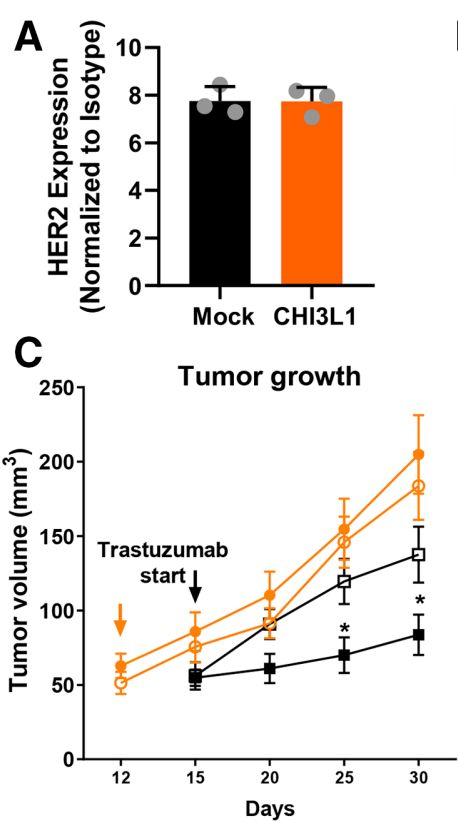

E

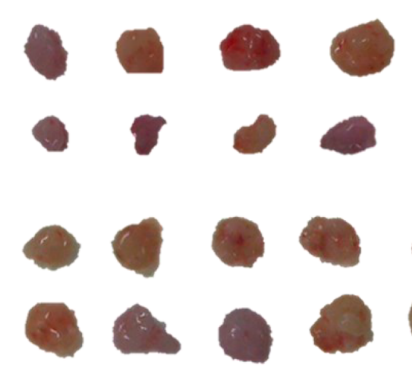

G

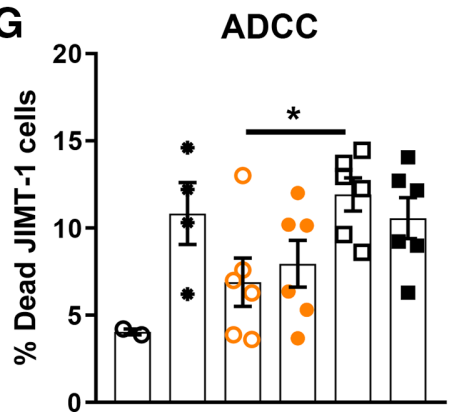

B

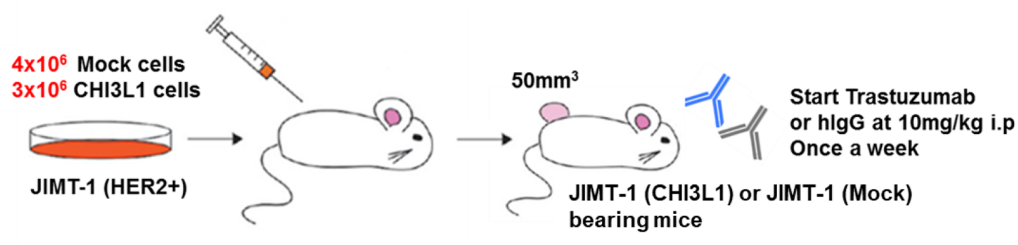

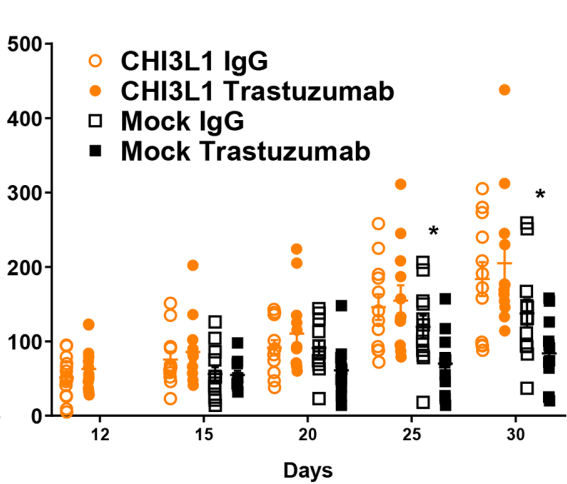

D Tumor weight
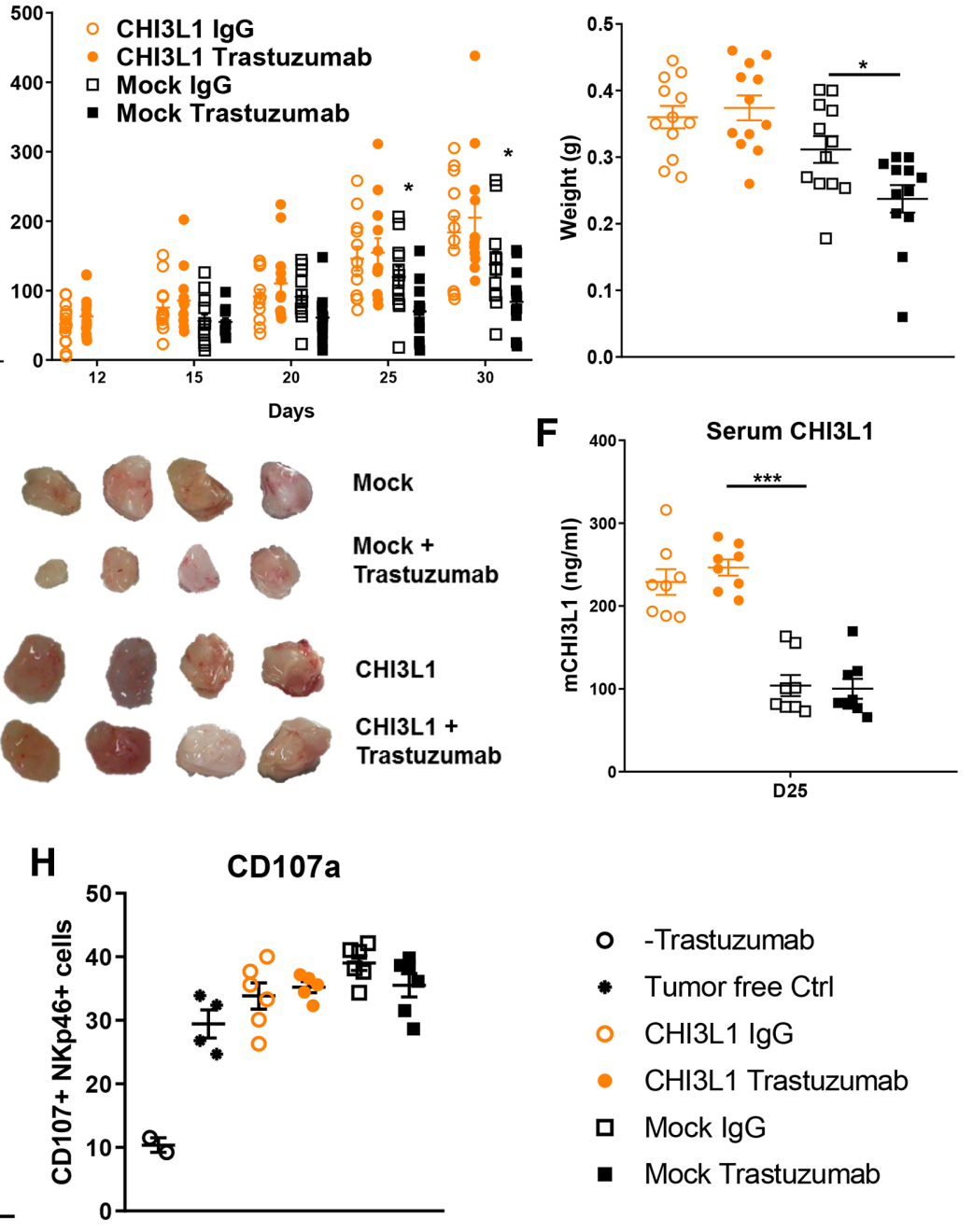

Figure 6 CHI3L1 overexpression abrogates the efficacy of Trastuzumab ADCC to control JIMT-1 tumors. (A) FACS analysis of HER2 expression on JIMT-1 mock and mCHI3L1-overexpressing cells stained with trastuzumab or hlgG (B). Experimental scheme. Tumor growth (C) and weight (D) in Trastuzumab or hlgG-treated mice are represented. (E) Representative images of tumors from different groups. (F) ELISA measurement of CHI3L1 sera levels. (G) Ex vivo ADCC assay against JIMT-1 cells using isolated splenocytes from mice of the indicated-experimental groups, cultured O.N with $10 \mathrm{ng} / \mathrm{mL} \mathrm{IL-15}$ and $200 \mathrm{UI} / \mathrm{mL} \mathrm{IL}-2$, (Effector:Target 40:1). (H) Measurement of degranulation by FACS analysis of CD107a staining by NKp46 ${ }^{+}$cells from the ex vivo cytotoxicity assay. Data are pooled from two independent experiments ( $\mathrm{N}=6$ mice per group for each). Values represent the means \pm SEM, $95 \% \mathrm{Cl},{ }^{*} \mathrm{p}<0.05,{ }^{* \star *} \mathrm{p}<0.001$ ). ADCC, antibody-dependent cellular cytotoxicity; CHI3L1, chitinase 3-like 1; IL-15, interleukin.

While IFN-g expression was induced similarly in Mock and CHI3L1 tumors on trastuzumab treatment, perforin induction was strongly abrogated with a similar trend for CD137, CD25 and T-bet expression (online supplemental figure S9E).

Altogether, these results indicate that CHI3L1 inhibits NK cell ADCC in vivo and may be responsible for resistance to trastuzumab treatment.

\section{A CHI3L1 neutralizing antibody synergizes with NK cell ADCC to cure tumor-bearing mice}

Our results have so far highlighted the inhibitory role of CHI3L1 on NK cell cytotoxicity, and as a direct consequence, its negative impact on Trastuzumab efficacy. This finding suggested that inhibiting this protein could potentially synergize with ADCC-mediating therapeutic antibodies to favor their efficacy. To test this hypothesis, 
A

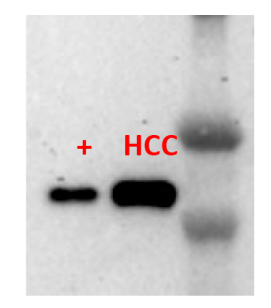

B

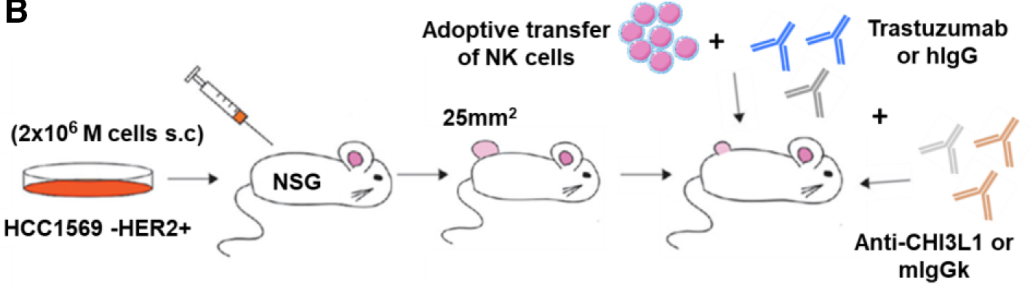

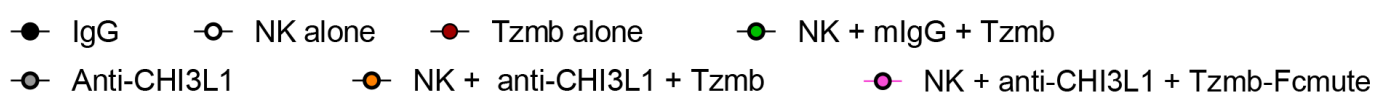

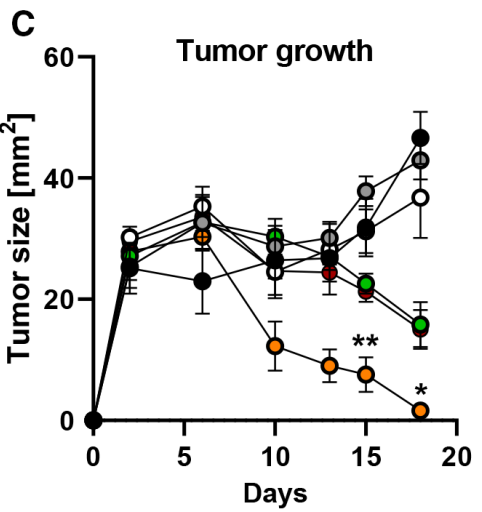

D

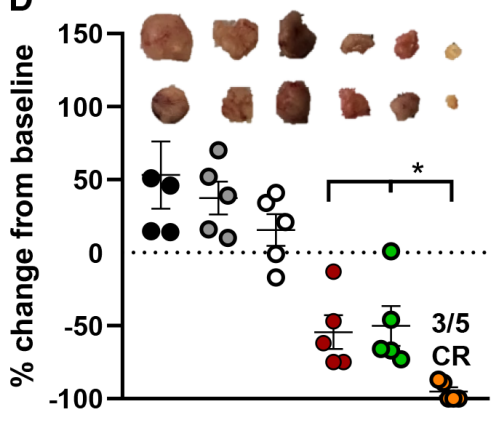

E

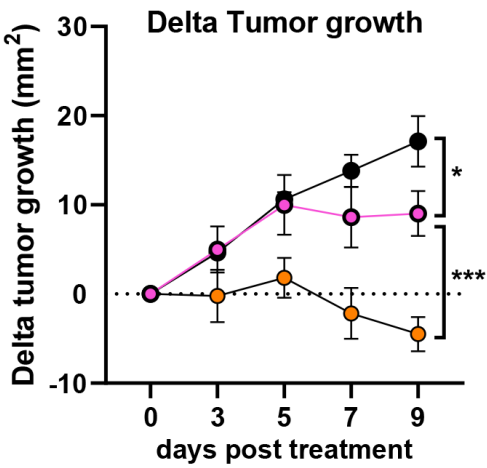

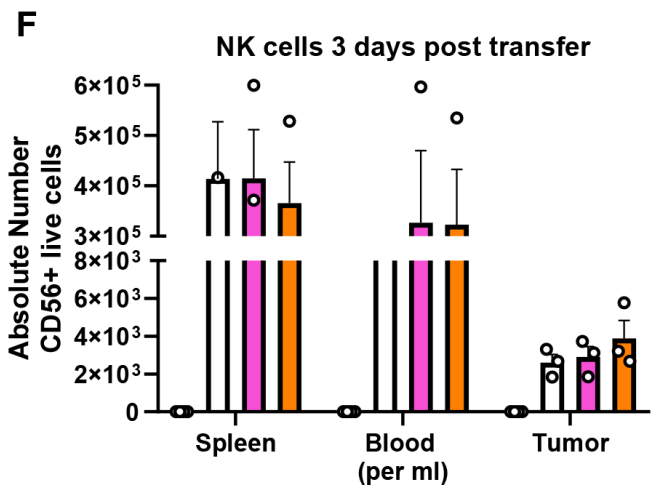

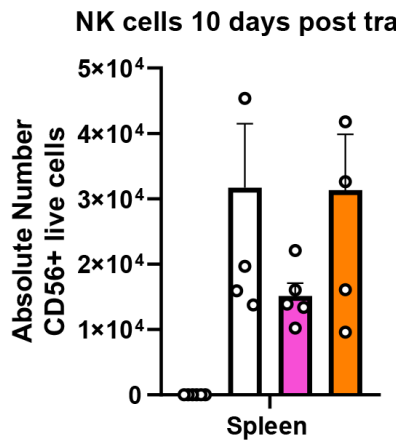
Untreated
K alone
NK + anti-CHI3L1
+ Tzmb-Fcmute
NK + anti-CHI3L1
+ Tzmb

Figure 7 Neutralizing CHI3L1 boosts the efficacy of Trastuzumab by unleashing NK cells. (A) Western blot image of hCHI3L1 from supernatant of HCC1569 cells ('+'=rhCHI3L1). (B) HCC1569 cells were injected in the flank of NSG mice. Once tumors reached $25 \mathrm{~mm}^{2}$ mice were assigned into groups of $\mathrm{N}=5$ mice and $8 \times 10^{6}$ isolated and expanded human NK cells were transferred via the tail vein into the indicated experimental groups (day 0). Anti-human $\mathrm{CHI} 3 \mathrm{~L} 1$ treatment started on day -1 $(200 \mu \mathrm{g} /$ mouse every 2 days) and trastuzumab on day $1(200 \mu \mathrm{g} /$ mouse once a week) of NK cell transfer. Tumor growth (C) and change from baseline (D) of different treatment groups. In (D) representative tumor images are shown above the tumor size variation for each experimental group. (E) Experiment was repeated with an Fc silent Trastuzumab, shown is variation of tumor growth from treatment start in each group (F) Enumeration of total $\mathrm{CD}_{56}{ }^{+}$human NK cells by FACS analysis among live splenocytes, in $1 \mathrm{~mL}$ of blood, or tumor lysates obtained from mice of the indicated experimental groups. Values represent the means \pm SEM, $95 \% \mathrm{Cl},{ }^{*} \mathrm{p}<0.05,{ }^{* *} \mathrm{p}<0.01$. CHI3L1, chitinase 3-like 1; NK, natural killer.

we used the human HCC1569 HER2 ${ }^{+}$breast cancer tumor cells that endogenously express high levels of human CHI3L1 (figure 7A). To reconstruct a relevant humanized therapeutic setting, we injected these tumor cells subcutaneously in NSG mice followed by transfer via the tail vain of ex vivo expanded healthy human NK cells. Mice were then subjected to treatment with Trastuzumab alone or in combination with an antihuman CHI3L1 neutralizing antibody, or its isotype control (figure 7B). We observed partial tumor control by Trastuzumab alone. This effect likely occurred via its direct action on the proliferation of HCC1569 HER2 $^{+}$cancer cells as human NK cell transfer did not have any additional benefit to Trastuzumab alone (figure 7C,D). This is presumably because of the effect of CHI3L1 on NK cells. Indeed, when mice were treated with a CHI3L1 neutralizing antibody, NK cell transfer in combination with trastuzumab led to a complete regression of the tumors (figure 7C,D). To highlight the involvement of NK cell ADCC in the observed clearance of tumors, we used an Fc- silent trastuzumab in combination with NK cells and anti-CHI3L1. This significantly reduced the ability of the triple combination to control tumor growth (figure 7E). Of note, NK cell persistence in the spleens, blood, and tumors was not affected by 
treatment 3 days after transfer and was low by the end of the experiment (figure 7F). These results demonstrate the therapeutic potential of targeting CHI3L1 to restore NK cell activity and boost trastuzumab (ADCC) efficacy in a robust preclinical model closely mimicking the human disease.

\section{DISCUSSION}

Major interest in the biology of chitinase-like proteins has been promoted by the family member CHI3L1 as its dysregulated expression has been linked to an array of human pathologies, including cancer. ${ }^{11}$ Our project aimed to study whether CHI3L1, which we found to be elevated in sera from trastuzumab resistant breast cancer patients, could promote tumor escape by protecting tumor cells from being killed by effector cytotoxic cells. Studies using different tumor models have shown that CHI3L1 overexpression leads to increased tumor growth and metastasis by inducing angiogenesis, proliferation, and resistance to apoptosis. ${ }^{11}$ In parallel, CHI3L1 has been shown to promote a type 2 immune response which is known to contribute to tumor development. ${ }^{49}$ Indeed, CHI3L1 KO mice show reduced Th2 inflammation, and overexpression of CHI3L1 reverses this phenotype. ${ }^{50}$ Conversely, ablation of CHI3L1 from murine T cells was shown to elicit a strong antimetastatic Th1 response with increased IFN- $\gamma$, perforin, and granzyme. ${ }^{19}$ Although these studies identified CHI3L1 as a significant immune modulator, a direct effect of this protein on cytotoxic cells such as NK cells in cancer patients was not yet characterized, nor was its precise mechanism of action.

Interestingly, dysfunction of the CTL, and more recently, of the NK cell IS has been described. ${ }^{4251}$ But the mechanism behind this especially within the TME remains elusive. In these studies, the inability of CTL and NK cells to polarize their granule content is related to insufficient signaling during synapse formation (due to lack of activation ligands on some targets) and could be corrected in vitro by cytokine treatment or the use of ADCC inducing antibodies, respectively. In our study, we describe a new mechanism of IS dysfunction mediated by CHI3L1 that affects both cytotoxic modalities (innate or ADCC) even after cytolytic commitment. We show through a series of in vitro, in vivo, and ex vivo experiments how CHI3L1mediated paralysis of the lytic granule machinery can lead to immune evasion and treatment resistance while targeting it can potentiate immunotherapy. Our findings could explain-at least in part-why immunotherapy efforts focused on targeting checkpoint receptors involved in earlier phases of the antitumor response (priming and recognition) fail to attain full clinical benefit.

IL13R $\alpha 2$ is a central receptor for CHI3L1 and mediates a broad range of its effects, ${ }^{35}$ however, in human NK cells, we observed only an intracellular expression of this receptor. This excluded its possible involvement in the activity of exogenous CHI3L1. By contrast, we observed that RAGE, another receptor of CHI3L1, was expressed on NK cells. There are two reports on RAGE function in NK cells to date, both highlighting an activating function of this receptor. ${ }^{39} 40$ Here we show that CHI3L1 behaves like a blocking agent for RAGE and inhibits its downstream JNK signaling, which is a key event for MTOC polarization. ${ }^{43}$ Interestingly, Parodi et al showed that several cytoskeletal or cytoskeleton-associated proteins were upregulated following RAGE ligation by HMGB1 in NK cells. ${ }^{40}$ In accordance, we found that RAGE accumulated at the IS, which is characterized by a major cytoskeletal rearrangement needed for the correct delivery of lytic granules into target cells. Altogether, these results highlight a new function of RAGE in controlling the cytoskeleton and granule biology in NK cells, and consequently their migration and cytotoxicity. This is relevant considering that diaphonous-1 (Dia-1), the signaling adaptor of RAGE, directly interacts with and regulates F-actin and tubulin structures. ${ }^{53}$ Importantly, the loss of DIA-1 in NK cells impairs MTOC polarization but without affecting synapse formation and F-actin accumulation. ${ }^{54}$ This closely resembles our observation on CHI3L1-mediated effects through RAGE. It would be interesting to know whether CHI3L1 also affects DIA-1 accumulation at the synapse during NK cell cytotoxicity.

NK cell ADCG is a major determinant of efficacy and patient responses to some monoclonal antibodies such as cetuximab and trastuzumab. ${ }^{4}$ To assess the precise role of CHI3L1 on NK cell ADCC, we employed two xenograft models of HER2 ${ }^{+}$breast cancer. In the first model, murine CHI3L1 overexpression rendered JIMT-1 tumors insensitive to trastuzumab treatment. This indicated a dysfunction of NK cells in mice bearing murine CHI3L1-overexpressing tumors, as Trastuzumab efficacy is solely dependent on NK cell ADCC in this model and not on inhibiting tumor cell proliferation. ${ }^{48}$ NK ADCC activity was indeed defective when tested ex vivo. In the second model, we established a therapeutic setting that would allow us to assess the potential of combining CHI3L1 blockade with Trastuzumab treatment. We used the human HCC1569 HER2 $^{+}$cell line, endogenously expressing high levels of human CHI3L1. Intriguingly, these cells were derived from a treatment-resistant patient but still partially responded to Trastuzumab-mediated HER2 inhibition in vitro. ${ }^{55}$ To reconstruct a relevant humanized therapeutic setting, we injected these cells in NSG mice followed by the transfer of human healthydonor isolated NK cells. The presence of NK cells did not have any additional benefit to Trastuzumab alone in these mice, suggesting that NK cells are inhibited due to a CHI3L1 rich environment. Strikingly, the addition of a human CHI3L1 neutralizing antibody to NK cell transfer and Trastuzumab led to complete regression of tumors. These results demonstrate that inhibiting CHI3L1 can restore NK cell activity and boost Trastuzumab (ADCC) efficacy in a robust preclinical model closely mimicking the human disease.

Important for the clinical relevance of this work, is our finding that sera from two cohorts of trastuzumab 
resistant patients contained high levels of CHI3L1 and inhibited NK cell ADCC which was reduced by a CHI3L1 neutralizing antibody further supporting the translational potential of our findings. The promising results obtained by Margetuximab (ADCC-enhanced version of Trastuzumab) ${ }^{7}$ and by Cetuximab in combination with the anti-NKG2A Monalizumab, ${ }^{8}$ support boosting NK cell activity to increase patient responses to anti-tumor antibodies. Interestingly, in some patients from our cohorts, we found that higher CHI3L1 levels preceded the clinical diagnosis of progression. Given the high expression of CHI3L1 in many cancer types, it would not qualify as an adequate and specific biomarker for HER2 breast cancer. Nevertheless, the variation of CHI3L1 levels within the same patient could be used to monitor clinical response, and this warrants investigation in larger patient cohorts.

Very recently, two studies associated HER2 ${ }^{+}$breast cancer with CHI3L1. One report showed that CHI3L1 facilitates migration and invasion of HER $2^{+}$tumor cells by stimulating epithelial to mesenchymal transition. ${ }^{56}$ The other showed that CHI3L1 is overexpressed in the cerebrospinal fluid of patients experiencing breast to brain metastasis (most frequent site in HER2 ${ }^{+}$breast cancer) and that inhibiting CHI3L1 impairs this process. ${ }^{57}$ These and our findings on the HER2-CHI3L1 axis come many years after the discovery of the murine CHI3L1/BRP-39 protein as one of the most overexpressed proteins in tumor cells derived from HER2 ${ }^{+} /$neu oncogene activation. ${ }^{58}$ They suggest that CHI3L1 may play several nonmutually exclusive protumorigenic functions and is an ideal target for HER2 ${ }^{+}$breast cancer treatment.

Besides ADCG, CHI3L1 can also inhibit the natural cytotoxicity of NK cells. This inhibition of NK cell tumor surveillance was revealed in vivo by the drastic increase in tumor engraftment and tumor size in the RMA-S NK-sensitive model following CHI3L1 injections. The similarity of this result to that obtained by NK cell depletion, and the lack of impact CHI3L1 has on RMA-S proliferation in vitro, would argue that the phenotype we observed is due to NK cell inhibition and in particular the NKG2Dperforin pathway. ${ }^{46}$

Despite this, we could not completely exclude other direct or indirect pro-tumorigenic effects of CHI3L1 in this model. Indeed, a combination of NK cell depletion and CHI3L1 injection show at least an additive effect on enhancing tumor growth suggesting an effect beyond that on NK cells. Similarly, in the previously discussed JIMT-1 model, CHI3L1 overexpression alone led to a marked increase in tumor size in inbred and outbred nude mice, despite not affecting cell proliferation in vitro. These findings are reminiscent of the well-documented protumorigenic properties of this protein (eg, angiogenesis),${ }^{16}$ to which we would add the important contribution of its immunosuppressive effects. Indeed, in outbred mice (more hostile to xenografts), we could appreciate the immune phenotype as mock tumors regressed but the CHI3L1 overexpressing ones continued to grow concomitantly with a decrease in NK cell activation. Moreover, we also observed that CHI3L1 (directly or indirectly) decreased cytotoxic granules of NKT and CD8 T cells in vivo as well as remarkably increasing macrophage infiltration into the tumors. Thus, based on data from these models, it is safe to say that CHI3L1 mediates its effects, at least in part, by shutting down NK-mediated immune surveillance, while also acting on other key immune cells in the TME which warrants further investigation.

In conclusion, our data demonstrate a new role of CHI3L1 as a functionally distinct soluble immune checkpoint molecule that acts by impairing the cytotoxic machinery. CHI3L1 acts on two fronts in mediating cancer progression. On one hand, its identification as a putative oncogene highlights the benefits it bestows on the tumor cell side. On the other hand, we show its deleterious effects on the anti-tumor immune response by NK cells and potentially other effector cells like CD8 T cells. Thus, the premise of targeting CHI3L1 will be to simultaneously inhibit an important molecule for tumor cell survival while enhancing antitumor immunity. Therefore, a combination of CHI3L1 inhibition with trastuzumab or other immunotherapeutics could be a highly promising approach to prevent treatment resistance and increase the number of responders.

\section{MATERIALS AND METHODS \\ Cell lines}

The different cell lines used in this work and their culture method are shown in online supplemental table S1.

Cell lines were purchased from the American Type Culture Collection. JIMT-1 cells were transfected with a PcDNA3.4 vector encoding murine CHI3L1 or with a mock vector (GeneArt, Life Technologies) and were selected with $400 \mu \mathrm{g} / \mathrm{mL}$ G418 for stable expression. Expi-CHO cell line was purchased from Thermo Fisher Scientific and handled according to the manufacturer's instructions. These cells were used for in-house production of recombinant CHI3L1. The cell lines used in experiments were regularly checked every 4 weeks for mycoplasma species with the commercial testing kit MycoAlert (Lonza).

\section{Primary cells}

Human PBMCs were isolated from buffy coats of healthy donors by ficoll density gradient separation using ficollplaque (GE Healthcare). Untouched primary NK cells or $\mathrm{CD}^{+} \mathrm{T}$ cells were negatively selected by magnetic separation using the $\mathrm{NK}$ cell and $\mathrm{CD} 8^{+} \mathrm{T}$ cell purification kits (Miltenyi Biotech, Bergisch Gladbach, Germany). Cells used in different assays had $>93 \%$ purity. NK and $\mathrm{CD} 8^{+}$ $\mathrm{T}$ cells were then cultured with RPMI 1640 (Life Technologies) supplemented with $10 \%$ heat-inactivated fetal bovine serum, $20 \mathrm{mM}$ glutamine, $1 \mathrm{mM}$ sodium pyruvate, 1:100 non-essential amino acids (Life Technologies). Before cytotoxicity assays, NK and $\mathrm{CD}^{+} \mathrm{T}$ cells were cultured overnight with 100UI/ml rhIL-2 (Peprotech) and then treated or not with different concentrations 
of rhCHI3L1 (RnD systems or produced in house). For murine NK cells, single-cell suspensions of murine spleens were prepared, and red blood cells were lysed with ammonium chloride lysis buffer. NK cells were negatively selected using the murine NK cell isolation kit (MiltenyiBiotec) and cultured in (Peprotech) $10 \mathrm{ng} /$ mL IL-15 and 100UI/ml IL-2 (Peprotech). On day 3, NK cell cultures with $>90 \%$ purity were treated or not with rmCHI3L1 (Sino biological or produced in house) for 1 hour before incubation with SKBR3 targets in ADCC or K562 targets for innate/natural cytotoxicity.

For the therapeutic experiment in NSG mice requiring NK cell adoptive transfer in combination with trastuzumab, primary human NK cells isolated as described above were activated and expanded for 2 weeks to reach sufficient numbers using the Miltenyi Biotec expansion and activation kit according to manufacturer protocol (cat\#130-094-483, Miltenyi biotec Bergisch Gladbach, Germany). Briefly, NK cells were cultured in NK MACS medium supplemented with $500 \mathrm{UI} / \mathrm{ml} \mathrm{IL}-2$ and $5 \mu \mathrm{L} / 10^{6}$ NK cells of MACS beads coated with MACS anti-CD2/ Anti-NKp46 agonistic antibodies. Using this protocol NK cells can be expanded at least 30-40 folds while retaining high viability.

\section{Patients}

Women with histological diagnosis of inoperable locally advanced or metastatic invasive breast cancer were considered eligible for the study if classified as HER-2 positive, that is, IHC score 3+or IHC score 2+and FISH amplified. We excluded enrollment of HER-2 positive patients with ER or PgR receptor-positive disease to avoid interference of hormonal therapy in our study.

Response to therapy was evaluated based on clinical, pathological, and radiologic examination of disease burden before and after treatment. The revised RECIST criteria (V.1.1) was used to evaluate the treatment response and patients were classified as responders in case of CR, partial response (PR) or SD or non-responders in case of progressive disease (PD).

- In the discovery cohort (2011-2013): A total of 20 patients were enrolled and underwent a first-line chemotherapy in combination with trastuzumab. Blood samples were collected at basal (before trastuzumab therapy) and subsequently until patients reached $\mathrm{PD}, \mathrm{SD}$ (12 months from enrollment), or CR state.

- In the validation cohort (2018-2020): Nine patients were enrolled in this study and received trastuzumab plus docetaxel and pertuzumab as first-line treatment followed by maintenance with trastuzumab +pertuzumab based on the conclusion and recommendations of the CLEOPATRA study. Blood was collected during response (at the time of proven control of disease) and considered as reference (basal time point), and at the time of change of clinical outcome to PD or SD/ PR/CR (within 18 months from enrolment).
Written informed consent was obtained from all patients.

\section{Animal experimentation}

Mice were purchased from Charles River Laboratories and were cared for and used under specific pathogenfree conditions according to the guidelines established in the Principles of Laboratory Animal Care (directive $86 / 609 /$ EEC).$^{59}$ Online supplemental table S2 summarizes the use of mice and cell lines in tumor experiments. The detailed experimental procedure can be found in the results section as a scheme before each experiment.

Tumor cells at $70 \%$ confluence were detached, washed twice, and resuspended in cold PBS, and kept on ice during the injection procedure. Tumor measurements and endpoints were registered by an observer blinded to the treatment groups. Tumor size $(>1.5 \mathrm{~cm})$ and necrosis, more than $15 \%$ wt loss or a decrease in general health condition (judged by decreased mobility, general weakness, hunched posture, or ungroomed hair) are defined as humane surrogate endpoints for survival and are later referred to as survival of mice.

\section{Cytotoxicity assay}

$\mathrm{NK}$ cell and $\mathrm{CD}^{+} \mathrm{T}$ cell-mediated cytotoxicity in vitro was determined using the DELFIA EuTDA Cytotoxicity Reagents (PerkinElmer Life Sciences, Waltham, Massachusetts, USA), according to manufacturer's instructions. Briefly, target cells were labeled with BATDA (a fluorescence enhancing ligand) in culture medium for $30 \mathrm{~min}$ at $37^{\circ} \mathrm{C}$ and then washed. Next, labeled target cells were transferred into a V-bottom sterile plate. In ADCC assays, SKBR3 cells were treated with $10 \mu \mathrm{g} / \mathrm{mL}$ Trastuzumab (Roche) for $20 \mathrm{~min}$ at $37^{\circ} \mathrm{C}$. In $\mathrm{CD} 8^{+} \mathrm{T}$ cells cytotoxicity assays, MEC1 targets were loaded with $2 \mu \mathrm{g} / \mathrm{mL}$ SEB sAg (SIGMA) for $30 \mathrm{~min}$ at $37^{\circ} \mathrm{C}$. Effector cells were treated as described in each experiment and then co-cultured with target cells in a v-bottom plate for $1 \mathrm{~h} 30 \mathrm{~min}$ in case of ADCC and 2 hours in case of natural NK cytotoxicity or $\mathrm{CD}^{+} \mathrm{T}$ cells cytotoxicity (effector/target ratio of 5:1). After incubation, $20 \mu \mathrm{L}$ of supernatant from each well was transferred to $180 \mu \mathrm{L}$ of europium (Eu) solution and the fluorescence was measured by time-resolved fluorometry (Victor3, PerkinElmer). The percent of specific release (=specific lysis) was calculated using the formula (experimental release - spontaneous release)/(maximum release - spontaneous release) $\times 100(\%)$.

In neutralization experiments, anti-CHI3L1 (clone MaY) (EMD Millipore) or mIgG1 Isotype were preincubated with the rhCHI3L1 or patients' sera for 1 hour at $37^{\circ} \mathrm{C}$. The mixture was then added to NK cells and incubated for 1 hour before coculture with target cells. In RAGE blocking experiments, NK cells were treated with anti-RAGE antibody, Isotype control, or rage antagonistic peptide overnight before cytotoxicity experiments.

When using splenocytes for ex-vivo cytotoxicity assays, killing activity was measured using a FACS-based readout 
of the percentage of dead targets cells (by live/dead stain) after coculture for the indicated times in each assay.

\section{Confocal microscopy}

For imaging NK cells alone, cells were treated as described and then cultured on poly-L-lysine coated slides (Thermo fisher scientific) for 2 hours at $37^{\circ} \mathrm{C}$ in a humidified chamber. For imaging the IS, NK cells and target cells were co-incubated at an E:T ratio of 2:1 for $15 \mathrm{~min}$ in a v-bottom plate to allow conjugate formation. Conjugates were then gently resuspended and deposited on poly-L-lysine coated slides and incubated in a humidified chamber for $30 \mathrm{~min}$ at $37^{\circ} \mathrm{C}$. Cells were then fixed and permeabilized for $10 \mathrm{~min}$ at RT in cytofix/cytoperm solution (BD bioscience), washed, and then blocked for 10 min with PBS, $10 \%$ FBS, in perm buffer. The indicated primary antibodies were added to the slide in blocking buffer for $30 \mathrm{~min}$ at RT or 18 hours at $4^{\circ} \mathrm{C}$. Slides were mounted with Vectashield H1000 mounting medium with DAPI (Vectorlabs) and imaged on Leica TCS SP8 system (Leica-microsystems) using $60 \times / 1.40 \mathrm{NA}$ oil objective. Image analysis and quantifications were carried out using National Institutes of Health ImageJ software.

Blinded confocal imaging and conjugate quantification were carried out following the selection of 10 representative areas of each slide. Cells in or out of conjugate within each area were quantified and a ratio thereof subsequently determined. For each conjugate, the position of the MTOC was observed, and its polarization to the IS, or lack thereof, was noted. The ratio of polarized to nonpolarized MTOCs was used to determine the ratio of functional synapses out of all conjugates formed.

\section{Live cell imaging}

$5 \times 10^{4}$ SKBR3 cells were seeded in a $\mu$-Slide 8 Well Glass Bottom overnight (Ibidi GmbH, Martinsried, Germany). $10^{5} \mathrm{NK}$ cells were washed once with phenol red-free RPMI-1640 medium, supplemented $20 \mathrm{mM}$ HEPES (Sigma Aldrich), $2 \mathrm{mM}$ L-glutamine, and non-essential amino acids (Life Technologies) and stained with SiRtubulin and verapamil (Spirochrome) $(500 \mathrm{nM}$ and $10 \mu \mathrm{M}$, respectively) for 1 hour at $37^{\circ} \mathrm{C}$. LysoTracker Red DND-99 (Life technologies) was then added to NK cells $(10 \mu \mathrm{M})$ for another $30 \mathrm{~min}$ at $37^{\circ} \mathrm{C}$. Following incubation, NK cells were washed three times and treated or not with $100 \mathrm{ng} / \mathrm{mL}$ CHI3L1 for 1 hour. In parallel, SKBR3 targets were treated with $10 \mu \mathrm{g} / \mathrm{mL}$ Trastuzumab for 20 min. After mounting the slide with the SKBR3 target cells, the medium containing Trastuzumab was removed before the addition of NK cells. Eight fields per condition were recorded for 2 hours with 180 s intervals. Live imaging was performed at $37^{\circ} \mathrm{C}$ using environmental chambers on a Leica SD AF Spinning Disc Confocal (Leica-microsystems).

\section{Flow cytometry and phosphoflow experiments}

Single-cell suspensions were incubated with FcR-blocking antibody before staining with primary antibodies (except for CD16 staining). Cell doublets were excluded by comparison of side-scatter width to forward-scatter area. Dead cells were excluded by live/dead staining using fixable live/dead stain dyes (Thermofisher). NK cells were gated as singlets, FSC-.SSC appropriate for lymphocytes, CD ${ }_{\text {neg }}$, CD56 $6_{\text {pos }}$ cells (as shown below). Intracellular staining was performed using a fixation and permeabilization buffer (BD Bioscience).

For phosphoflow experiments, NK cells were fixed with $4 \%$ PFA for $12 \mathrm{~min}$ at $37^{\circ} \mathrm{C}$ immediately after treatment ends. Cells were then permeabilized with ice cold methanol-based Perm-III buffer (BD Biosciences) for $30 \mathrm{~min}$ on ice. Cells were then washed three time with FACS buffer to eliminate residual and stained with directly conjugated phosphospecific antibodies (BD Biosciences). Cells were analyzed on FACSCanto II cytometer (BD Biosciences). Flow cytometry data were analyzed with FlowJo v10 software (Tree Star).

\section{ELISA}

Human and murine CHI3L1 levels in different sample types (patient and mice sera) were measured using ELISA kits (R\&D systems) according to the manufacturer's instructions. Blood was always processed in less than 2 hours after collection and was kept at $4^{\circ} \mathrm{C}$ to avoid nonspecific release of CHI3L1 from neutrophils.

Absorbance was measured at $450 \mathrm{~nm}$ using Clariostar Plate reader (BMG Labtech).

\section{Western blot}

The endogenous human CHI3L1 secreted from HCC1569 cells or the murine CHI3L1 secreted by transfected JIMT-1 cells were detected by western blot analysis of supernatant from $70 \%$ confluent culture $(20 \mu \mathrm{L})$ using goat antihuman CHI3L1 polyclonal antibody (R\&D systems) and rat anti-mouse antibody (R\&D systems), respectively.

\section{Cell proliferation}

The CyQUANT Cell Proliferation Assay (Thermofisher Scientific) was used to determine the effect of CHI3L1 treatment or overexpression on the proliferation of tumor cells in vitro before conducting tumor experiments with these cells. Cells were plated at $1 \times 10^{4}$ cells/well in 96 well flat-bottom plates. Some plates were harvested the same day, a time when very little proliferation had occurred and considered as T0. Parallel culture plates, either control or treated, were then harvested after 48 hours or 72 hours. The assay was conducted according to the manufacturer's instruction and acquired with a matrix-based scanning acquisition of each well on Clariostar reader (BMG Labtech).

\section{Cohorts' analysis (public databases)}

Gene expression data were obtained from the R2 Genomics Analysis and Visualization Platform (http:// r2.amc.nl) or TCGA database and CHI3L1 expression data were median centered per array and plotted with PRISM (GraphPad Software) or using the GEPIA plotter (http://gepia.cancer-pku.cn) (for TCGA data vs normal 
breast). The univariate survival analysis of different patient cohorts was performed using the $\mathrm{R}^{2}$ KaplanMeier analysis module or Kmplotter (http://kmplot. com) which included comprehensive information on the relevant clinical and prognostic factors selected for analysis. Probesets in each database with the highest average signals were selected for analysis.

\section{Statistics}

The statistical significance between the two groups was determined with unpaired Student's t-test, whereas the comparison of multiple groups was carried out by oneway or two-way ANOVA, followed by Bonferroni's post-test for correction of multiple comparisons using GraphPad Prism software (San Diego, California, USA). The univariate survival analysis of different breast cancer and gastric cancer cohorts was performed using the $\mathrm{R}^{2}$ Kaplan-Meier analysis module or Kmplotter (http://kmplot.com) both based on the log-rank test. The cut-off was defined to yield the most significant (likelihood ratio test) split separation with a minimum group size of 30 . A $p \leq 0.05$ was considered to denote significance.

\section{Antibodies}

A list of all antibodies used is provided in online supplemental table S3.

\section{Author affiliations}

${ }^{1}$ Department of Biomedical Sciences, Humanitas University, Milan, Italy

${ }^{2}$ RRCCS Humanitas Research Hospital, via Manzoni 56, 20089, Rozzano, Milan, Italy

${ }^{3}$ Department of Medicine IV, Ludwig-Maximilians-Universitat Munchen, Munchen,

Bayern, Germany

${ }^{4}$ Division for Clinical Pharmacology, Ludwig-Maximilians-Universitat Munchen,

Munich, Germany

${ }^{5}$ European Institute of Oncology, IRCCS, Milano, Italy

${ }^{6}$ Roche Innovation Center Munich, Penzberg, Germany

${ }^{7}$ Universita degli Studi di Milano, Milano, Italy

${ }^{8}$ Division of Clinical Pharmacology, Ludwig-Maximilians-Universitat Munchen, Munich, UK

Correction notice This article has been updated since it was first published. Affiliation 2 has been updated and affiliation 5 has been added to author Giuseppe Curigliano.

\section{Twitter Bruno Cadilha @bcadilha}

Contributors Conceptualization: AD, AS, GP, JM, and M-RB; methodology: AD, AS, JM, GP, CS and MR; investigation: AD, AS, M-RB, AM, BC, DS, HO, AL and LP; validation: $M-R B, B C, H O$ and $S K$; writing-original draft: $A D$; writing-review and editing: M-RB, GP, SK, BC, CS and MR; funding acquisition: $A D$ and MR; resources: GC, GM, SK and AT; supervision: CS, GP and MR;guarantor: AD and MR.

Funding This study was supported by the Marie-Sklodowska-Curie Program Training Network for the Immunotherapy of Cancer funded by the H2020 Program of the European Union (Grant 641549 to MR and SK), the AIRC investigator's grant (ref. 22026 to MR), the Marie-Sklodowska-Curie Program Training Network for Optimizing Adoptive T cell therapy (Grant 955575 to SK), funded by the H2020 Program of the European Union, the International Doctoral Program i-Target: Immunotargeting of Cancer funded by the Elite Network of Bavaria (SK) and the European Research Council Starting Grant (grant number 756017 to SK).

Competing interests None declared.

\section{Patient consent for publication Not applicable.}

Ethics approval This study was approved by the Ethics Committee of the European Institute of oncology hospital, Milan, Italy. This study was approved by the Ethics Committee of Humanitas clinical institute, Milan, Italy.
Provenance and peer review Not commissioned; externally peer reviewed.

Data availability statement Data sharing not applicable as no datasets generated and/or analysed for this study. Data are available uon reasonable request. All data relevant to the study are included in the article or uploaded as online supplemental information.

Supplemental material This content has been supplied by the author(s). It has not been vetted by BMJ Publishing Group Limited (BMJ) and may not have been peer-reviewed. Any opinions or recommendations discussed are solely those of the author(s) and are not endorsed by BMJ. BMJ disclaims all liability and responsibility arising from any reliance placed on the content. Where the content includes any translated material, BMJ does not warrant the accuracy and reliability of the translations (including but not limited to local regulations, clinical guidelines, terminology, drug names and drug dosages), and is not responsible for any error and/or omissions arising from translation and adaptation or otherwise.

Open access This is an open access article distributed in accordance with the Creative Commons Attribution Non Commercial (CC BY-NC 4.0) license, which permits others to distribute, remix, adapt, build upon this work non-commercially, and license their derivative works on different terms, provided the original work is properly cited, appropriate credit is given, any changes made indicated, and the use is non-commercial. See http://creativecommons.org/licenses/by-nc/4.0/.

\section{ORCID iDs}

Abbass Darwich http://orcid.org/0000-0001-5540-9303

Sebastian Kobold http://orcid.org/0000-0002-5612-4673

\section{REFERENCES}

1 Mandal A, Viswanathan C. Natural killer cells: in health and disease. Hematol Oncol Stem Cell Ther 2015;8:47-55.

2 Orange JS. Formation and function of the lytic NK-cell immunological synapse. Nat Rev Immunol 2008;8:713-25.

3 Bhat R, Watzl C. Serial killing of tumor cells by human natural killer cells--enhancement by therapeutic antibodies. PLoS One 2007;2:e326.

4 Tamura Ket al. Fc $\gamma R 2 A$ and $3 \mathrm{~A}$ polymorphisms predict clinical outcome of trastuzumab in both neoadjuvant and metastatic settings in patients with HER2-positive breast cancer. Ann Oncol 2011;22:1302-7.

5 Beano A, Signorino E, Evangelista A, et al. Correlation between NK function and response to trastuzumab in metastatic breast cancer patients. J Trans/ Med 2008;6:25.

6 Kute TE, Savage L, Stehle JR, et al. Breast tumor cells isolated from in vitro resistance to trastuzumab remain sensitive to trastuzumab anti-tumor effects in vivo and to ADCC killing. Cancer Immunol Immunother 2009;58:1889-98.

7 Rugo HS, Im S-A, Wright GLS, et al. SOPHIA primary analysis: a phase $3(P 3)$ study of margetuximab (M) + chemotherapy $(C)$ versus trastuzumab $(T)+C$ in patients (pts) with HER2+ metastatic (met) breast cancer (MBC) after prior anti-HER2 therapies (Tx). JCO 2019;37:1000.

8 Cohen RB, Lefebvre G, Posner MR, et al. Monalizumab in combination with cetuximab in patients (pts) with recurrent or metastatic (R/M) head and neck cancer (SCCHN) previously treated or not with PD-(L)1 inhibitors (IO): 1-year survival data. Ann Oncol 2019;30:v460.

9 Recklies AD, White $\mathrm{C}$, Ling $\mathrm{H}$. The chitinase 3-like protein human cartilage glycoprotein 39 (HC-gp39) stimulates proliferation of human connective-tissue cells and activates both extracellular signal-regulated kinase- and protein kinase B-mediated signalling pathways. Biochem J 2002;365:119-26.

10 Sutherland TE. Chitinase-like proteins as regulators of innate immunity and tissue repair: helpful lessons for asthma? Biochem Soc Trans 2018;46:141-51.

11 Zhao T, Su Z, Li Y, et al. Chitinase-3 like-protein-1 function and its role in diseases. Signal Transduct Targeted Therapy 2020;5.

12 Erturk K, Tas F, Serilmez M, et al. Clinical significance of serum Ykl-40 (chitinase-3-like-1 protein) as a biomarker in melanoma: an analysis of 112 Turkish patients. Asian Pac J Cancer Prev 2017;18:1383-7.

13 Faibish M, Francescone R, Bentley B, et al. A YKL-40-neutralizing antibody blocks tumor angiogenesis and progression: a potential therapeutic agent in cancers. Mol Cancer Ther 2011;10:742-51.

14 Qiu Q-C, Wang L, Jin S-S, et al. CHI3L1 promotes tumor progression by activating TGF- $\beta$ signaling pathway in hepatocellular carcinoma. Sci Rep 2018;8:15029. 
15 Wan G, Xiang L, Sun X, et al. Elevated YKL-40 expression is associated with a poor prognosis in breast cancer patients. Oncotarget 2017;8:5382-91.

16 Zhang J-P, Yuan H-X, Kong W-T, et al. Increased expression of chitinase 3 -like 1 and microvessel density predicts metastasis and poor prognosis in clear cell renal cell carcinoma. Tumour Biol 2014;35:12131-7.

17 Darr C, Krafft U, Hadaschik B, et al. The role of YKL-40 in predicting resistance to docetaxel chemotherapy in prostate cancer. Urol Int 2018;101:65-73.

18 Cohen N, Shani O, Raz Y, et al. Fibroblasts drive an immunosuppressive and growth-promoting microenvironment in breast cancer via secretion of chitinase 3-like 1. Oncogene 2017;36:4457-68.

19 Kim D-H, Park H-J, Lim S, et al. Regulation of chitinase-3-like-1 in T cell elicits Th1 and cytotoxic responses to inhibit lung metastasis. Nat Commun 2018;9.

20 Geng B, Pan J, Zhao T, et al. Chitinase 3-like 1-CD44 interaction promotes metastasis and epithelial-to-mesenchymal transition through $\beta$-catenin/Erk/Akt signaling in gastric cancer. J Exp Clin Cancer Res 2018;37.

21 Ma B, Herzog EL, Moore M, et al. RIG-like helicase regulation of chitinase 3-like 1 axis and pulmonary metastasis. Sci Rep 2016;6:26299.

22 Park K-R, Yun H-M, Yoo K, et al. Chitinase 3 like 1 suppresses the stability and activity of p53 to promote lung tumorigenesis. Cell Commun Signal 2020;18:5.

23 Abd El-Fattah AA, Sadik NAH, Shaker OG, et al. Single nucleotide polymorphism in SMAd7 and CHI3L1 and colorectal cancer risk. Mediators Inflamm 2018;2018:9853192

24 Shaker OG, Helmy HS. Circulating bone-related markers and YKL-40 versus HER2 and TOPO2a in bone metastatic and nonmetastatic breast cancer: diagnostic implications. Clin Breast Cancer 2018;18:e321-8.

25 López-Soto A, Gonzalez S, Smyth MJ, et al. Control of metastasis by NK cells. Cancer Cell 2017;32:135-54.

26 Stojanovic A, Cerwenka A. Natural killer cells and solid tumors. J Innate Immun 2011;3:355-64.

27 Wang W, Erbe AK, Hank JA, et al. NK cell-mediated antibodydependent cellular cytotoxicity in cancer immunotherapy. Front Immunol 2015;6:368.

28 Hu W, Wang G, Huang D, et al. Cancer immunotherapy based on natural killer cells: current progress and new opportunities. Front Immunol 2019;10:1205.

29 Tamura K, Shimizu C, Hojo T, et al. Fc $\gamma$ R2A and 3A polymorphisms predict clinical outcome of trastuzumab in both neoadjuvant and metastatic settings in patients with HER2-positive breast cancer. Ann Oncol 2011;22:1302-7.

$30 \mathrm{He} \mathrm{W}$, Tong Y, Wang Y, et al. Serum soluble CD14 is a potential prognostic indicator of recurrence of human breast invasive ductal carcinoma with Her2-enriched subtype. PLoS One 2013;8:e75366.

31 Breit SN, Johnen H, Cook AD, et al. The TGF- $\beta$ superfamily cytokine, MIC-1/GDF15: a pleotrophic cytokine with roles in inflammation, cancer and metabolism. Growth Factors 2011;29:187-95.

$32 \mathrm{He} \mathrm{L}, \mathrm{Li} \mathrm{J}$, Zhan J, et al. The value of serum cystatin C in early evaluation of renal insufficiency in patients undergoing chemotherapy: a systematic review and meta-analysis. Cancer Chemother Pharmacol 2019;83:561-71.

33 Lattanzio L, Denaro N, Vivenza D, et al. Elevated basal antibodydependent cell-mediated cytotoxicity (ADCC) and high epidermal growth factor receptor (EGFR) expression predict favourable outcome in patients with locally advanced head and neck cancer treated with cetuximab and radiotherapy. Cancer Immunol Immunother 2017;66:573-9.

34 Maréchal R, De Schutter J, Nagy N, et al. Putative contribution of CD56 positive cells in cetuximab treatment efficacy in first-line metastatic colorectal cancer patients. BMC Cancer 2010;10:340.

$35 \mathrm{He} \mathrm{CH}$, Lee CG, Dela Cruz CS, et al. Chitinase 3 -like 1 regulates cellular and tissue responses via IL-13 receptor $\alpha 2$. Cell Rep 2013;4:830-41.

36 Low D, Subramaniam R, Lin L, et al. Chitinase 3-like 1 induces survival and proliferation of intestinal epithelial cells during chronic inflammation and colitis-associated cancer by regulating S100A9. Oncotarget 2015;6:36535-50.
37 Zhou Y, He CH, Herzog EL, et al. Chitinase 3-like-1 and its receptors in hermansky-pudlak syndrome-associated lung disease. J Clin Invest 2015;125:3178-92.

38 Chen Y, Perussia B, Campbell KS. Prostaglandin D2 suppresses human NK cell function via signaling through $\mathrm{D}$ prostanoid receptor. $J$ Immunol 2007;179:2766-73.

39 Narumi K, Miyakawa R, Ueda R, et al. Proinflammatory proteins S100A8/S100A9 activate NK cells via interaction with RAGE. $\mathrm{J}$ Immunol 2015;194:5539-48.

40 Parodi M, Pedrazzi M, Cantoni C, et al. Natural Killer (NK)/melanoma cell interaction induces NK-mediated release of chemotactic High Mobility Group Box-1 (HMGB1) capable of amplifying NK cell recruitment. Oncoimmunology 2015;4:e1052353.

41 Xie J, Méndez JD, Méndez-Valenzuela V, et al. Cellular signalling of the receptor for advanced glycation end products (RAGE). Cell Signal 2013;25:2185-97.

42 Kabanova A, Zurli V, Baldari CT. Signals controlling lytic granule polarization at the cytotoxic immune synapse. Front Immunol 2018;9:307

$43 \mathrm{Li} \mathrm{C}, \mathrm{Ge} \mathrm{B}$, Nicotra M, et al. JNK MAP kinase activation is required for MTOC and granule polarization in NKG2D-mediated NK cell cytotoxicity. Proc Natl Acad Sci U S A 2008;105:3017-22.

44 Libreros S, Garcia-Areas R, Shibata Y, et al. Induction of proinflammatory mediators by $\mathrm{CHI} 3 \mathrm{~L} 1$ is reduced by chitin treatment: decreased tumor metastasis in a breast cancer model. Int $J$ Cancer 2012;131:377-86.

45 Ljunggren H. -G, Öhlén C, Höglund P. The RMA-S lymphoma mutant; consequences of a peptide loading defect on immunological recognition and graft rejection. Int J Cancer 1991:47:38-44.

46 van den Broek MF, Kägi D, Zinkernagel RM, et al. Perforin dependence of natural killer cell-mediated tumor control in vivo. Eur J Immunol 1995;25:3514-6.

47 Daniel V, Sadeghi M, Wang H. CD4 +CD25 +Foxp3 +IFN- $\gamma$ + human induced $\mathrm{T}$ regulatory cells are induced by interferon- $\gamma$ and suppress alloresponses nonspecifically. Hum Immunol 2011.

48 Barok M, Isola J, Pályi-Krekk Z, et al. Trastuzumab causes antibodydependent cellular cytotoxicity-mediated growth inhibition of submacroscopic JIMT-1 breast cancer xenografts despite intrinsic drug resistance. Mol Cancer Ther 2007;6:2065-72.

49 Colotta F, Allavena P, Sica A, et al. Cancer-related inflammation, the seventh hallmark of cancer: links to genetic instability. Carcinogenesis 2009;30:1073-81.

50 Lee CG, Hartl D, Lee GR, et al. Role of breast regression protein 39 (BRP-39)/chitinase 3-like-1 in Th2 and IL-13-induced tissue responses and apoptosis. J Exp Med 2009;206:1149-66.

51 Eitler J, Wotschel N, Miller N, et al. Inability of granule polarization by NK cells defines tumor resistance and can be overcome by CAR or ADCC mediated targeting. J Immunother Cancer 2021;9.

52 Chen X, Trivedi PP, Ge B, et al. Many NK cell receptors activate ERK2 and JNK1 to trigger microtubule organizing center and granule polarization and cytotoxicity. Proc Natl Acad Sci U S A 2007;104:6329-34

53 Bershadsky AD, Ballestrem C, Carramusa L, et al. Assembly and mechanosensory function of focal adhesions: experiments and models. Eur J Cell Biol 2006;85:165-73.

54 Butler B, Cooper JA. Distinct roles for the actin nucleators Arp2/3 and hDia1 during NK-mediated cytotoxicity. Curr Biol 2009;19:1886-96.

55 Jernström S, Hongisto V, Leivonen S-K, et al. Drug-screening and genomic analyses of HER2-positive breast cancer cell lines reveal predictors for treatment response. Breast Cancer 2017:9:185-98.

56 Morera E, Steinhäuser SS, Budkova Z, et al. YKL-40/CHI3L1 facilitates migration and invasion in HER2 overexpressing breast epithelial progenitor cells and generates a niche for capillary-like network formation. Vitr Cell Dev Biol Anim 2019;55:838-53.

57 Ansari KI, Bhan A, Liu X, et al. Astrocytic IGFBP2 and CHI3L1 in cerebrospinal fluid drive cortical metastasis of HER2+breast cancer. Clin Exp Metastasis 2020;37:401-12.

58 Morrison BW, Leder P. Neu and Ras initiate murine mammary tumors that share genetic markers generally absent in c-myc and int-2initiated tumors. Oncogene 1994;9:3417-26.

59 Louhimies S. Directive 86/609/EEC on the protection of animals used for experimental and other scientific purposes. Altern Lab Anim 2002;30:217-9. 


\section{Correction: Paralysis of the cytotoxic granule machinery is a new cancer immune evasion mechanism mediated by chitinase 3-like-1}

Darwich A, Silvestri A, Benmebarek M-R, et al. Paralysis of the cytotoxic granule machinery is a new cancer immune evasion mechanism mediated by chitinase 3-like-1. J Immunother Cancer 2021;9:e003224. doi:10.1136/jitc-2021-003224

Affiliation 2 has been updated to: IRCCS Humanitas Research Hospital, via Manzoni 56, 20089 Rozzano, Milan, Italy

In addition to this, affiliation five has been added to author Giuseppe Curigliano.

Open access This is an open access article distributed in accordance with the Creative Commons Attribution Non Commercial (CC BY-NC 4.0) license, which permits others to distribute, remix, adapt, build upon this work non-commercially, and license their derivative works on different terms, provided the original work is properly cited, appropriate credit is given, any changes made indicated, and the use is non-commercial. See http://creativecommons.org/licenses/by-nc/4.0/.

(C) Author(s) (or their employer(s)) 2022. Re-use permitted under CC BY-NC. No commercial re-use. See rights and permissions. Published by BMJ.

J Immunother Cancer 2022;10:e003224corr1. doi:10.1136/jitc-2021-003224corr1

A) Check for updates 